\title{
The environmental test campaign of GMOD: a novel gamma-ray detector
}

Joseph Mangan, David Murphy, Rachel Dunwoody, Alexey Ulyanov, Joseph Thompson, et al.

Joseph Mangan, David Murphy, Rachel Dunwoody, Alexey Ulyanov, Joseph Thompson, Umair Javaid, Conor O'Toole, Maeve Doyle, Masoud Emam, Jessica Erkal, Gianluca Fontanesi, Jack Kyle, Fergal Marshall, Rakhi Rajagopalan Nail, Favour Okosun, Jack Reilly, Sarah Walsh, Daithí de Faoite, Lána Salmon, Lorraine Hanlon, David McKeown, William O'Connor, Kenneth Stanton, Ronan Wall, Brian Shortt, Joost Vanreusel, David Palma, Lily Ha, Sheila McBreen, "The environmental test campaign of GMOD: a novel gamma-ray detector," Proc. SPIE 11852, International Conference on Space Optics - ICSO 2020, 1185214 (11 June 2021); doi: 10.1117/12.2599225

SDIE Event: International Conference on Space Optics - ICSO 2021, 2021, Online Only 


\section{International Conference on Space Optics-ICSO 2020}

Virtual Conference

30 March-2 April 2021

Edited by Bruno Cugny, Zoran Sodnik, and Nikos Karafolas
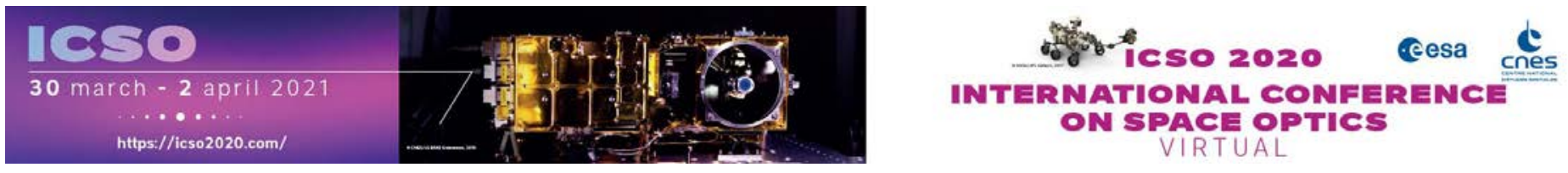

\section{The environmental test campaign of GMOD: a novel gamma-ray detector}

\section{Cesa issopoceatings lecnes}




\title{
The Environmental Test Campaign of GMOD: a Novel Gamma-Ray Detector
}

Joseph Mangan ${ }^{\mathrm{a}}$, David Murphy ${ }^{\mathrm{a}}$, Rachel Dunwoody ${ }^{\mathrm{a}}$, Alexey Ulyanov ${ }^{\mathrm{a}}$, Joseph Thompson ${ }^{\mathrm{b}}$, Umair Javaid $^{\mathrm{b}}$, Conor O'Toole ${ }^{\mathrm{c}}$, Maeve Doyle ${ }^{\mathrm{a}}$, Masoud Emam ${ }^{\mathrm{b}}$, Jessica Erkal ${ }^{\mathrm{a}}$, Gianluca Fontanesi $^{\mathrm{d}}$, Jack Kyle ${ }^{\mathrm{a}}$, Fergal Marshalle,a, Rakhi Rajagopalan Nair ${ }^{\mathrm{b}}$, Favour Okosun ${ }^{\mathrm{b}}$, Jack Reilly $^{\mathrm{a}}$, Sarah Walsh ${ }^{\mathrm{a}}$, Daithí de Faoite ${ }^{\mathrm{b}}$, Lána Salmon ${ }^{\mathrm{a}}$, Lorraine Hanlon ${ }^{\mathrm{a}}$, David McKeown ${ }^{\mathrm{b}}$, William O'Connor ${ }^{\mathrm{b}}$, Kenneth Stanton ${ }^{\mathrm{b}}$, Ronan Wall ${ }^{\mathrm{a}}$, Brian Shortt ${ }^{\mathrm{f}}$, Joost Vanreusel ${ }^{\mathrm{f}}$, David Palma $^{\mathrm{g}}$, Lily $\mathrm{Ha}^{\mathrm{h}}$, and Sheila McBreen ${ }^{\mathrm{a}}$

a School of Physics, University College Dublin, Ireland

${ }^{\mathrm{b}}$ School of Mechanical and Materials Engineering, University College Dublin, Ireland

'School of Mathematics and Statistics, University College Dublin, Ireland

${ }^{\mathrm{d} S c h o o l ~ o f ~ E l e c t r i c a l ~ a n d ~ E l e c t r o n i c ~ E n g i n e e r i n g, ~ U n i v e r s i t y ~ C o l l e g e ~ D u b l i n, ~ I r e l a n d ~}$

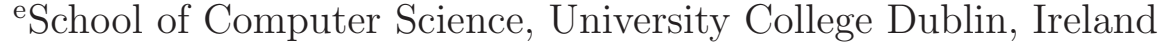

${ }^{\mathrm{f}}$ European Space Agency, ESTEC, Netherlands

'Redu Space Services for European Space Agency, ESEC, Redu, Belgium

${ }^{\mathrm{h}} \mathrm{HE}$ Space Operations for European Space Agency, ESTEC, Noordwijk, Netherlands

\begin{abstract}
The Gamma-Ray Module (GMOD) is a novel gamma-ray detector developed for the study of high energy astrophysical transients called Gamma-Ray Bursts. GMOD has been designed in-house and will be flown on board EIRSAT-1, intended to be Ireland's first satellite, a 2U CubeSat developed as part of the European Space Agency's Fly Your Satellite! programme. The detector comprises a $25 \times 25 \times 40 \mathrm{~mm} \mathrm{CeBr}_{3}$ scintillator, coupled to a tiled array of 16 OnSemiconductor Silicon Photomultipliers with front-end readout provided by the IDE3380 SIPHRA. The readout is received by the GMOD Motherboard which provides temporary storage and support functionality for the instrument operation, including the transfer of Time-Tagged Event data to the EIRSAT-1 On Board Computer. The Engineering Qualification Model was environmentally tested following an approach tailored from the ECSS standards in early February 2020 at the CubeSat Support Facility in Transinne, Belgium. This campaign was conducted to qualify the hardware for low Earth orbit, including multi-axis vibration testing and thermal-vacuum cycling under qualification test levels and durations. GMOD was mounted on a $20 \mathrm{kN}$ electrodynamic shaker in which it underwent predefined sine and random vibration test profiles, demonstrating its ability to withstand the launch environment. The instrument was then thermally cycled under vacuum over

a range spanning $-31^{\circ} \mathrm{C}$ to $+75^{\circ} \mathrm{C}$, simulating the low Earth orbit environment. The results of the successful environmental qualification campaign of the Gamma-Ray Module from EIRSAT-1 are presented.
\end{abstract}

Keywords: Gamma-ray Detectors, CubeSats, Space Qualification, Gamma-Ray Bursts

\section{INTRODUCTION}

The Gamma-Ray Module (GMOD) is a novel, CubeSat compatible gamma-ray detector developed for the detection of extremely energetic cosmic events, called Gamma-Ray Bursts (GRBs). ${ }^{1,2}$ These events are characterised by a beamed emission of highly collimated gamma-ray photons which are detectable in Low Earth Orbit (LEO), lasting up to seconds to minutes in duration ${ }^{3}$ and which are associated with the death of massive stars ${ }^{4}$ and the merger of massive compact objects. ${ }^{5,6}$ The detection of GW170817, ${ }^{7}$ the gravitational wave signal from a binary neutron star merger, by the Laser Interferometer Gravitational-Wave Observatory (LIGO),, 8 in coincidence with

Further author information: E-mail: joseph.mangan@ucdconnect.ie, Telephone: +3531716 2202 
a short Gamma-Ray Burst detection, GRB170817A, ${ }^{10}$ was a breakthrough discovery beginning a new gravitational wave era and underlining the importance of gamma-ray missions to the future detection and localisation of GRBs in the context of multi-messenger astronomy. Currently there are a number of large operational missions with gamma-ray instrumentation including The Neil Gehrels Swift Telescope, ${ }^{11}$ Fermi Space Telescope ${ }^{12,13}$ and INTEGRAL. ${ }^{14}$ The sky coverage of these missions can be enhanced on relatively short timescales by the bespoke development of CubeSat missions designed for GRB detection, such as BurstCube. ${ }^{15}$ GMOD will be flown as a scientific payload on board The Educational Irish Research Satellite - 1 (EIRSAT-1), ${ }^{16}$ a 2U CubeSat under development with the European Space Agency (ESA) Fly Your Satellite! (FYS) programme. ${ }^{17}$ The payload will demonstrate the instrument's capabilities for detection of GRBs in LEO, using an instrument heritage ${ }^{18-20}$ which has been modified for CubeSat compatibility with a Commercial Off-The-Shelf (COTS) stack. Prototype hardware has been previously tested during a high altitude balloon flight from Palestine, Texas (Murphy et al. 2020, submitted) and during a $101.4 \mathrm{MeV}$ proton irradiation test of the detector assembly. ${ }^{21}$ The detector is comprised of a Cerium Bromide $\left(\mathrm{CeBr}_{3}\right)$ scintillator which is optically coupled to a custom built Silicon Photomultiplier (SiPM) array with front-end readout provided by the SIPHRA Application Specific Integrated Circuit (ASIC). SiPMs ${ }^{22}$ are currently not widely used in space applications as they are a relatively new technology, however they have a number of advantages, including small and easily integrable form factors, high gain, magnetic field immunity and low power and bias voltage requirements, making them a future alternative to traditional Photomultiplier Tube (PMT) based instruments. The high resolution $\mathrm{CeBr}_{3}{ }^{23}$ scintillator is also relatively new and a good spectral match with the SiPM array, being a less expensive alternative to Lanthanum Bromide $\left(\mathrm{LaBr}_{3}\right)$, allowing fast decay times and high light yield output. The IDE3380 SIPHRA ${ }^{24}$ ASIC is a 16-channel SiPM/Multi-Pixel Photon Counter (MPPC) readout solution which has been used in previous studies and was developed by IDEAS Norway, for the first time being environmentally qualified during this campaign. An in-house designed and built detector Motherboard (MB) in PC/104 form factor was custom built to provide power, detector readout/storage and support functionality for the instrument during operation.

GMOD will detect $\sim 18 \mathrm{GRBs}$ in a one year mission within the standard $50 \mathrm{keV}$ to $300 \mathrm{keV}$ energy range and will downlink spectra, light-curves and Time-Tagged Event (TTE) data (Murphy in prep, 2021). In this paper, the standard space qualification environmental tests conducted on the GMOD Engineering Qualification Model (EQM) are presented. This includes an overview of the instrument, the equipment and software used for data acquisition, vibration and thermal-vacuum test results and an overview of the detector's performance throughout.

\section{THE GAMMA-RAY MODULE}

The detector assembly is shown in Figure 1, consisting of a $25 \mathrm{~mm} \times 25 \mathrm{~mm} \times 40 \mathrm{~mm}$ Scionix $\mathrm{CeBr}_{3}$ crystal* $^{*}$ coupled to an array of 16 MicroFJ-60035-TSV SiPMs supplied by OnSemiconductor. The SiPMs are mounted in a tiled, $4 \times 4$ array configuration on a custom built Printed Circuit Board (PCB), which is coupled optically to the $\mathrm{CeBr}_{3}$ scintillator using an Eljen EJ-560 ${ }^{\dagger}$ silicone rubber interface. SiPM readout is performed using the the 16-channel IDE3380 Silicon Photomultiplier Readout ASIC (SIPHRA) ${ }^{24}$ which is supplied as a wire bonded die to a carrier Chip on Board $(\mathrm{CoB}) \mathrm{PCB}^{\ddagger}$. The final element of the detector electronics assembly is a custom built SiPM-toASIC PCB interface, which connects the SiPM array outputs to the ASIC, and the detector electronics to the GMOD motherboard.

The detector electronics assembly is loaded into a light-tight aluminium enclosure along the $\mathrm{X}$-axis of the GMOD Coordinate Reference Frame (CRF) (as seen in Figure 1), which is sealed at both ends with aluminium end-caps. Within the aluminium housing the scintillator and electronics assembly are secured using a set of PTFE spacers which support and prevent compression of the SiPM array and electronics components. PTFE inserts are placed about each corner of the scintillator with a final spacer being placed between the scintillator and the -X-axis end-cap. This aluminium end-cap features a $1 \mathrm{~mm}$ venting hole while the PTFE spacer has a

${ }^{*} \mathrm{The}_{\mathrm{CeBr}}$ crystal is wrapped in PolyTetraFluoroEthylene (PTFE) helping to improve scintillation light collection, it is then hermetically encased within its own aluminium housing, with a $2 \mathrm{~mm}$ thick quartz window for optical transmission. This process is preformed by Scionix as purchased.

${ }^{\dagger}$ URL: https://eljentechnology.com/products/accessories/ej-560

${ }^{\ddagger}$ URL: https://ideas.no/the-ide3380-asic-carrier-board/ 
serpentine venting channel to mitigate stray light ingress and release any trapped air while in vacuum conditions. The detector assembly is mounted onto the $+\mathrm{Z}$ face of the motherboard using a set of 6 M2 threaded cap screws.

The detector assembly is electronically mated with the GMOD MB by a set of DF13 connectors which connect to the SiPM-to-ASIC interface PCB and which are routed to the exterior of the detector assembly through slots in the $+\mathrm{X}$-axis aluminium end-cap (Figure 1, harnessing and slots not shown). These connections supply the detector assembly with power and provide a two way channel for configuration and transmission of event readout for processing by the MB. The MB supports the instrument operation through generation of regulated power and bias supply, TTE data preprocessing, readout and storage, ASIC and general configuration for the instrument operation. This is accomplished using a Xilinx XC2C256 CoolrunnerII Complex Programmable Logic Device (CPLD) and a Texas Instruments (TI) MSP430FR5994 micro-controller.

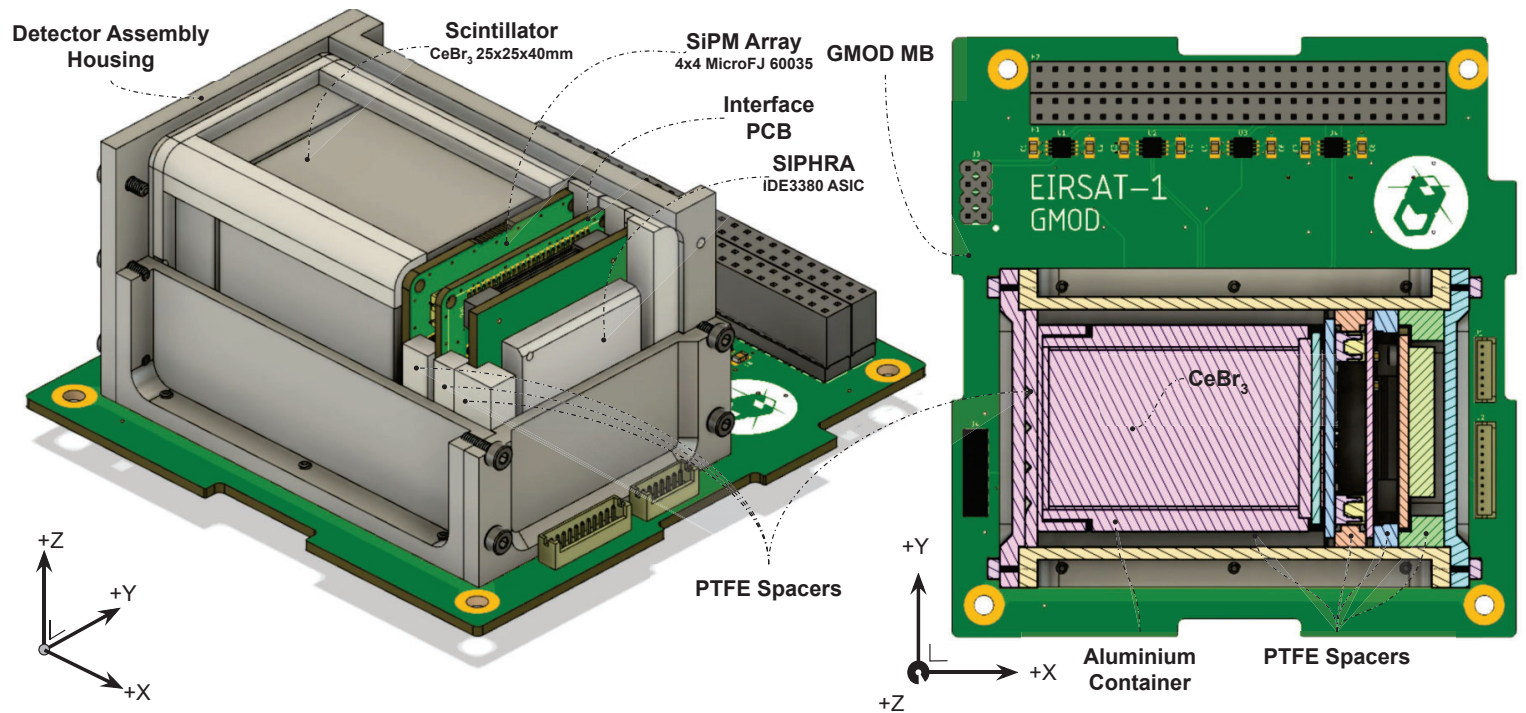

Figure 1: Left) A pealed back view of GMOD's internal electronics assembly, supported by PTFE spacers, showing the exposed $\mathrm{CeBr}_{3}$ scintillator, SiPM array PCB, interface PCB and ASIC CoB. Right) A top-down, + Z-axis view, with detector assembly cutaway showing internal assembly components.

SIPHRA $^{24}$ is capable of processing the analog signals from the SiPM array by use of a per-channel current integrator circuit with adjustable trigger threshold, gain, shaping and a track-and-hold system, enabling measurements for pulse height spectroscopy and 16-channel SiPM readout. The combined signal of the individual active channels can be used to produce a summed measurement output, while the SiPM array temperature may also be measured using a PT100 sensor mounted on the underside of the array PCB and sampled by SIPHRA at trigger time. Upon triggering a gamma-ray event, SIPHRA samples the peak heights and outputs a serialised data stream of 12 bit digitised 16-channel and summed pulse height measurements with PT100 channel readout. The event data must be immediately time-tagged for GRB triggering and optimised for efficient memory allocation and later manipulation by the GMOD MB (Mangan et al 2021, in prep). The data stream is transmitted to the CPLD which converts the raw data into TTE packets which are received by the MSP430, processed and temporally stored in $128 \mathrm{MBit}$ flash for later service to the satellite On Board Computer (OBC). ${ }^{25}$ The MSP430 also provides support functionality to the instrument, in particular controlling the bias voltage generation, SIPHRA configuration, management of the external flash memory and TTE data collection, storage and transmission upon request to the OBC. The operational layout diagram of GMOD is shown in Figure 2.

\section{QUALIFICATION AND ENVIRONMENTAL TEST APPROACH}

Between the $3^{\text {rd }}$ and the $7^{\text {th }}$ of February 2020 the GMOD EQM underwent environmental qualification testing at the ESA CubeSat Support Facility (CSF) in Belgium. The EQM hardware was vibration tested in all three axes, simulating the g-force effects imparted by the launch vehicle during launch ascent, followed by thermal-vacuum testing, simulating the LEO environment. The purpose of environmental testing can be clearly understood in 


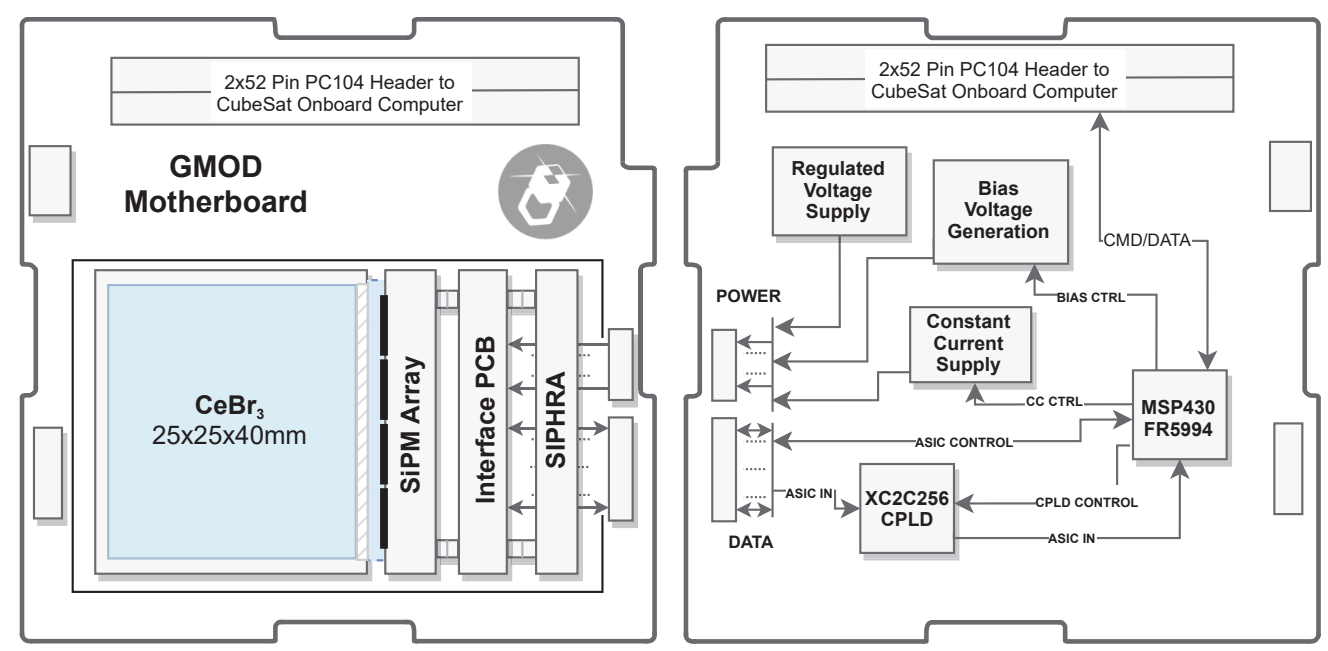

Figure 2: Left) Schematic operational layout diagram of GMOD, including scintillator and SiPM arrangements on the top of the MB. Right) The principal components for readout and control with flow of operation.

the context of the typical mission life cycle adopted by most space projects. ${ }^{26,27}$ These phases, outlined in ECSS-M-ST-10C, ${ }^{28}$ provide a structure which stages the development of a space mission from inception (Phase A: Feasibility) to end of life (Phase F: Disposal). System level environmental testing of the spacecraft falls under Phase D: Qualification and Production operations, concerning the production of EQM and Flight Model (FM) hardware and software (depending on mission model philosophy) and the completion of qualification and acceptance activities. However, it is common for subsystem level environmental testing and qualification to occur during Phase C: Detailed Definition and Phase D, concerning the development and qualification of space/ground segments and their products. ${ }^{28}$ In the case of GMOD, the EQM was subsystem level tested (results presented here) and will later be system level tested while integrated in the satellite stack.

The model philosophy (as discussed in ECSS-E-HB-10-10-02 $\mathrm{A}^{29}$ ) which is adopted by the mission dictates the classification of the various iterations of hardware developed (ie. demonstration models, engineering-qualification model, proto-flight model, flight spare, flight model), its purpose and the test levels which it may be subjected to during verification. In the case of EIRSAT- $1^{30}$ the "prototype" approach was taken which is tailored to missions developing novel payloads, previously untested and complex in design. Under the prototype philosophy a number of models are produced for the GMOD payload. The Demonstration Model (DM) acts as a functional equivalent of the flight model hardware allowing design feasibility and electrical/functional unit level testing during development. The EQM combines the conventional Engineering model (EM) and Qualification model (QM) definitions, describing a model which is representative of the form, fit and function of the flight model with some flexibility on the use of high reliability parts. This may be used for fit and functional testing, validation and environmental testing of the payload hardware. The GMOD EQM is the model which was used during the environmental testing campaign and is subjected to qualification test levels and durations as specified in ECSSE-ST-10-03. ${ }^{31,32}$ This was developed as an exact copy of the FM hardware including the use of the same high reliability components and PCB fabrication and assembly facilities. It is not intended for flight but, according to the prototype model philosophy, may later be used as a flight spare or for ground support testing during the mission life time. Finally, the FM is the final implementation reflecting the complete realised design intended for flight.

In general, verification is the process whereby the Item Under Test (IUT) is tested to prove that it has been designed and produced in agreement with the specifications (including any agreed deviations/wavers), that it is free from workmanship defects and conforms to the specified requirements and performance criteria intended for its mission life cycle. ${ }^{29}$ This is performed using a number of methods, with testing being preferred for higher confidence results. During qualification, environmental testing is performed on test articles which are flight representative, this provides confidence in the product's performance during launch and in the harsh conditions 
of the space environment. Following on from successful qualification, the results of the qualification stage are fed into the acceptance stage to assess whether the flight hardware is free from workmanship or manufacturing errors. This manner of verification was selected for the qualification of GMOD, independent from the rest of the spacecraft, as it was custom built and not COTS hardware. Furthermore, it would confirm the design and highlight any non-conformance which may not be apparent prior to the test campaign. The test levels, durations and margins are a function of the verification stage, model philosophy and level of hardware decomposition being tested. In this case, GMOD was tested as "space segment equipment" 31 with appropriate qualification test levels, durations and margins as agreed by ESA test facility coordinators.

A number of documents are produced in the lead up to, during and after testing. These documents are intended to be a guide through the test process as well as a record of the performance at each stage. The Test Specification (TSPE) and Test Procedure (TPRO) ${ }^{31}$ documents were merged into a single document; the Test Specification - Test Procedure (TSTP) which defines the purpose of test, an overview of the IUT, pass/fail requirements, the Ground Support Equipment (GSE) and the step by step procedures for conducting the test. The Test Readiness Review (TRR) report acts as a record of the TRR meeting held just prior to the test activity. This is to confirm that the conditions of the IUT, facility equipment and the program test levels are appropriately set up for the requirements outlined in the TSTP and to determine whether testing is ready to begin. Similarly, the Post Test Review (PTR) reports the PTR meeting and summarises the test operation, confirming whether the test was conducted in accordance with the TSTP and TRR specifications as well as discussing any nonconformances which may have occurred. The Test Report (TRPT) presents an in-depth discussion on the execution of the test campaign and provides an interpretation and analysis of the data collected. In the case of non-conformance and deviation, a Non-Conformance Report (NCR) (as defined in ECSS-Q-ST-10-09C ${ }^{33}$ ) documents the extent and nature of any anomalies observed with the IUT, GSE, test equipment as well as actions of test operators and technicians which were contrary to the specifications outlined in the TSTP and TRR. The NCR includes an overview of the root cause of the investigation and the corrective actions taken (if any). An NCR may lead to a Request for Waiver, if a satisfactory and agreed approach to continue has been found. Once testing and documentation have been complete, a Test Review Board (TRB) is called, a panel of all those involved which meet to close out the test activity and to approve the test report findings.

\subsection{Functionality, Firmware \& Data Acquisition,}

The MSP430 firmware used during the environmental tests was a development version which provided the basic capabilities necessary to run the experiment, to check the peripheral devices after vibration and during thermalvacuum testing and finally to enable communications for data acquisition via Inter-Integrated Circuit $\left(\mathrm{I}^{2} \mathrm{C}\right)$ and serial protocols. Similarly, a CPLD Hardware Description Language (HDL) firmware was prepared for the environmental tests which was in a comparatively more advanced stage of development.

A custom adaptor for command and data acquisition between GMOD and the Electrical Ground Support Equipment (EGSE) was built for the environmental campaign. The EGSE used included a set of $4 \mathrm{~mm}$ banana plugs and a 3 channel RS Pro SPD3303X-E Power Supply Unit (PSU), a Total Phase Aardvark used as a Universal Serial Bus (USB) to $\mathrm{I}^{2} \mathrm{C}$ adaptor and a FTDI LC234X module as a USB to serial interface. Command and data collection was performed using a laptop running Linux Ubuntu OS and a custom User Interface (UI) developed for collection of data from GMOD for spectral integration, SiPM temperature monitoring and telemetry logging. The UI was built using the Tkinter Python toolkit with functionality for command and data handling developed in Python3. The EGSE interfaced with GMOD through a pair of custom adaptor PCBs, one being fabricated for vacuum compatibility and mating directly with GMOD and the other featuring the EGSE breakout connections. This interface was designed such that it could be used for both the vibration and Thermal-Vacuum Chamber (TVAC) Reduced Functional Test (RFT) inspections allowing a D-Sub-37 electrical feedthrough between the DN200 ISO-K flange in the Small Space Simulator $\left(\mathrm{S}^{3}\right)$ TVAC wall. The EGSE layout including adaptors can be seen in Figure 3.

Standard calibration sources which are normally used during spectral integration and which produce known gamma-ray energy emissions were not available and would likely not be vacuum compatible. These were needed for functional testing, tests which consisted of a 10 minute spectral integration used to perform a general health check of the instrument and to roughly gauge any variation in performance. As a substitute, a set of $2 \%$ thoriated 


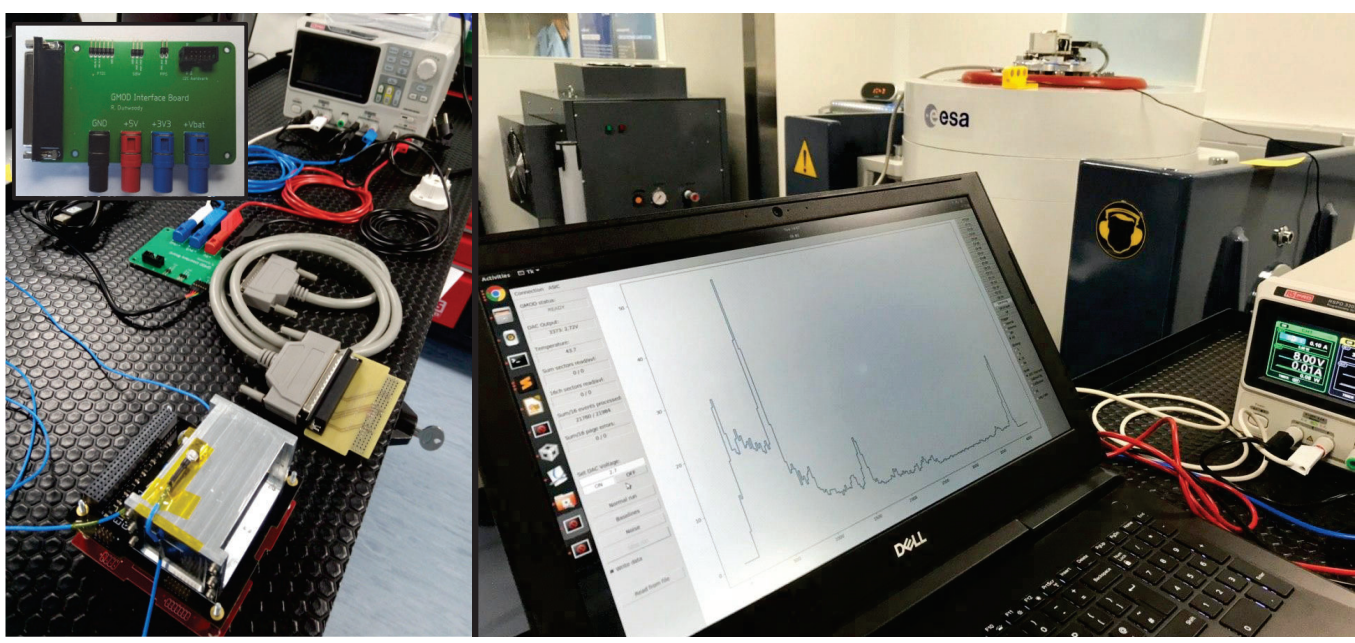

Figure 3: Left) GMOD and associated EGSE. Shown are the vacuum compatible TVAC boards with power and serial connections prepared for interfacing with GMOD, with the EGSE breakout board is shown in the inset. Right) The EGSE being used to record a spectrum while GMOD is mounted on the vibration table.

tungsten welding rods were used, containing the gamma-ray emitting radioisotope Thorium-232 in the form of Thorium oxide. When ${ }^{232} \mathrm{Th}$ and its daughter isotopes undergo radioactive decay, they produce alpha and beta ray emissions in addition to a number of known gamma-ray photons, producing a distinct gamma-ray spectrum with prominent peaks. The ${ }^{232} \mathrm{Th}$ spectrum was measured using a high resolution High Purity Germanium (HPGe) detector which is shown in Figure 4 with calibrated peak energies ${ }^{34}$ highlighted for reference. A set of 7 red tipped cylindrical rods $(1.6 \times 150 \mathrm{~mm})$ were procured, estimated to contain $1.7 \%-2.2 \%$ radioactive Thorium oxide per rod according to ISO standard $6848^{35}$ with a maximum activity of up to $\sim 7 \mathrm{kBq}^{36}(0.19 \mu \mathrm{Ci})$ and contact dose rate of $\sim 1 \mu \mathrm{Sv} \mathrm{h}{ }^{-1}$ with rapid fall off with inverse distance squared. These rods are particularly convenient as an alternative to standard gamma-ray sources, they are low activity allowing easy transport, allow relatively safe handling, are cheap and are commercially available.

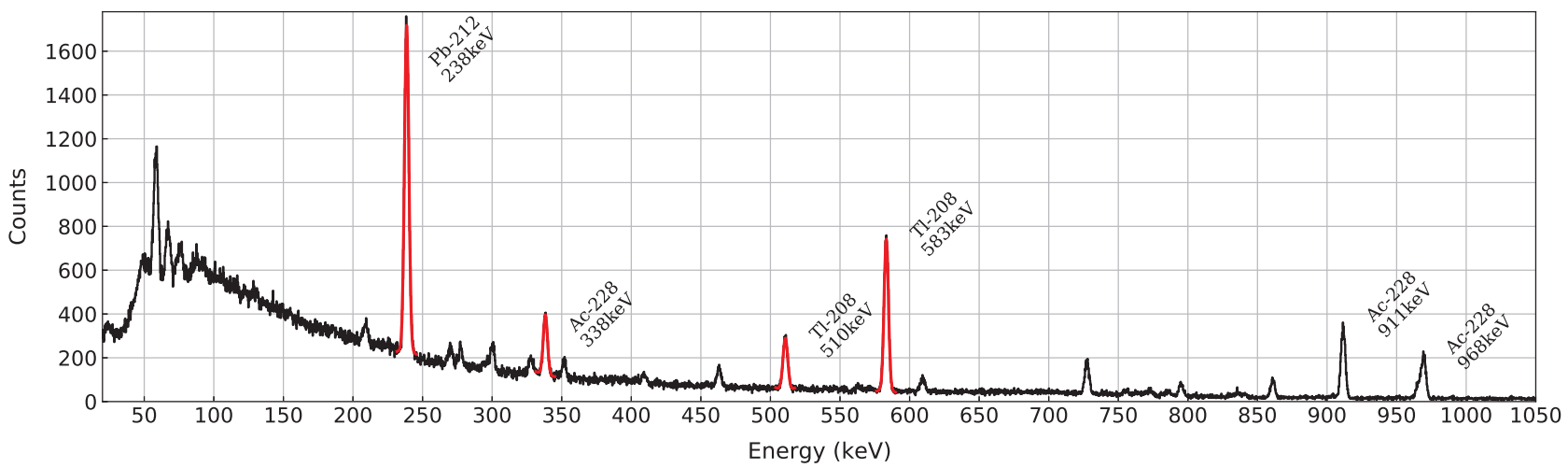

Figure 4: HPGe spectrum of the ${ }^{232} \mathrm{Th}$ containing welding rods calibrated with reference to the Idaho National Laboratory (INL) Catalogue https://gammaray.inl.gov/SitePages/Home.aspx of radioisotope spectra measured using $\mathrm{Ge}(\mathrm{Li})$ detectors. The four lines in solid red are the most relatively intense in the GMOD spectra and are readily identified for verification purposes during functional check.

\subsection{Vibration Testing}

\subsubsection{Modelling and Simulations}

A finite element analysis was performed on GMOD in order to verify that the structure of the payload can withstand the loads experienced during launch of the satellite. The EIRSAT-1 Computer Aided Design (CAD) 
Table 1: Quasi-static, sine and random vibration Von Mises stress and MoS calculations for the GMOD MB PCB. Note: for the random vibration values the V.M stress values are the $3 \sigma$ measurements.

\begin{tabular}{|c|c|c|c|c|c|c|c|}
\hline \multirow[t]{2}{*}{ Loading Case } & \multirow[t]{2}{*}{ Yield (MPa) } & \multicolumn{3}{|c|}{ Max. V.M. Stress (MPa) } & \multicolumn{3}{|c|}{ Margin of Safety } \\
\hline & & X Load & Y Load & Z Load & X Load & Y Load & Z Load \\
\hline Quasi-Static Acc. & 262 & 34.21 & 27.23 & 51.62 & 5.1 & 6.7 & 3.1 \\
\hline Sine Vibration & 262 & 5.208 & 4.204 & 8.939 & 39.2 & 48.9 & 22.4 \\
\hline Random Vibration. & 262 & 42.03 & 31.78 & 55.99 & 4.0 & 5.6 & 2.7 \\
\hline
\end{tabular}

model was used to develop a Finite Element (FE) model of the structure with Autodesk Inventor and Autodesk NASTRAN-in-CAD. ${ }^{37}$ The GMOD model was constrained by the PCB corner holes representing the mounting configuration while integrated within in the EIRSAT-1 stack. Normal Mode Analysis was performed on the GMOD FE model to calculate the natural frequencies and mode shapes of GMOD. For each mode, the modal effective masses in each Degree of Freedom (DOF) was determined, with modes $>10 \%$ being used to inform the placement of the accelerometers for the vibration campaign. A number of loading cases were then simulated in all three axes, including quasi-static acceleration, sine vibration and random vibration. These were used to determine the Margin of Safety (MoS) with respect to the yield stress calculated using the Von Mises stress in each material for a given loading direction. The MoS was calculated as the ratio between the design allowable stress and the design limit stress scaled by a factor of safety of 1.25 per ECSS-E-ST-32-10C (2009). ${ }^{38}$ Simulations show that while positive, the minimum calculated MoS (throughout the spacecraft) of 3.1, 22.4 and 2.7 occurred in the GMOD MB along the Z-axis direction during quasi-static, sine vibration and random vibration loading respectively. This reflects the effect of having a large concentrated mass such as the GMOD detector assembly mounted atop the MB PCB. The calculated MoS for the MB is shown in Table 1 for all three axes and loading cases.

\subsubsection{Test Levels and Set-Up}

Prior to any testing at the CSF, GMOD and accompanying EGSE were unpacked and cleaned with Isopropyl Alcohol (IPA). A visual inspection and functional check was then performed to ensure that no damage had occurred during transport. Three measurement accelerometers were installed on GMOD by the CSF operators. The position of the accelerometers were chosen to detect the primary modes during vibration as determined from the FE analysis described in Section 3.2.1. Accelerometers A1 and A2 were installed on the $+\mathrm{Z}$ face of the detector housing and the GMOD MB surface respectively (Figure 5A), while accelerometer A3 was placed on the $-\mathrm{Z}$ face of the MB surface (Figure 5B). GMOD was mounted to the shaker table via two custom machined aluminium mounting plates: the Shaker Interface Plate (SIP), which was mounted to the shaker, and the Subsystem Adaptor Plate (SAP), which mounted on the SIP and which interfaced GMOD to the shaker table (Figure 5C). Control accelerometers $\mathrm{C} 1$ and $\mathrm{C} 2$ were mounted on the SIP as close as possible to the mounting position of the instrument. GMOD was attached to the SIP via the four mounting holes on the MB. To replicate the flight configuration of the payload in the EIRSAT-1 PCB stack, 5mm PTFE spacers centred on the MB mounting holes were placed above and below the MB PCB. The mounting screws for the SAP and GMOD were marked with permanent marker during installation so any rotation could be observed after vibration. The spacers and screw markings can be seen in Figure 5 at each corner of the MB PCB. The levels and durations for the sine ${ }^{32}$ and random ${ }^{39}$ vibration tests, including tolerances, are detailed in Table 2. As the launch vehicle is yet to be determined (International Space Station (ISS) orbit presumed), the vibration test levels are selected based on qualifying the payload for a variety of launch providers such that they cover the minimum requirements for the JAXA H-II Transfer Vehicle (HTV), SpaceX Dragon or Northrop Grumman Orbital Cygnus spacecraft. ${ }^{40,41}$

\subsubsection{Vibration Test Operation \& Results}

Sine and random vibration tests were performed for each axis with respect to the instrument's CRF. Sine vibration was conducted as a sinusoidal sweep from 5 up to $100 \mathrm{~Hz}$ at 2 octaves per minute. The random vibration sequence was comprised of consecutive 10s intervals relative to the specified test levels and then a final interval of $120 \mathrm{~s}$ at $0 \mathrm{~dB}$. Resonance searches, consisting of a low level sine sweep, were performed before and after each sine and random vibration run. This was used to generate a frequency response of the structure which 
Table 2: Vibration test levels and durations for sine, random and resonance searches

\begin{tabular}{|c|c|c|c|c|}
\hline \multicolumn{5}{|c|}{ Sine Vibration Tests } \\
\hline \multicolumn{3}{|c|}{ Levels } & Duration & Tolerances \\
\hline Frequency $(\mathrm{Hz})$ & Value & Unit & \multirow{4}{*}{$\begin{array}{l}5-100 \mathrm{~Hz} \text { sweep } \\
@ 2 \text { oct } / \mathrm{min} \\
\approx 130 \mathrm{~s} \text { per axis }\end{array}$} & \multirow{4}{*}{$\begin{array}{cl}\text { Frequency: } & \pm 2 \% / \pm 1 \mathrm{~Hz} \\
\text { (Whichever is greater) } \\
\text { Amplitude: } \quad \pm 10 \% \\
\text { Sweep rate: } \quad \pm 5 \%\end{array}$} \\
\hline $5-21$ & 11 & $\mathrm{~mm}$ & & \\
\hline $21-60$ & 20 & & & \\
\hline $60-65$ & -2.8 & $\mathrm{~g} / \mathrm{Hz}$ & & \\
\hline \multicolumn{5}{|c|}{ Random Vibration Tests } \\
\hline \multicolumn{3}{|c|}{ Levels } & Duration & Tolerances \\
\hline Frequency $(\mathrm{Hz})$ & Value & Unit & \multirow{13}{*}{$\begin{array}{c}10 \mathrm{~s} \text { at }-9 \mathrm{~dB} \\
10 \mathrm{~s} \text { at }-6 \mathrm{~dB} \\
10 \mathrm{~s} \text { at }-3 \mathrm{~dB} \\
120 \mathrm{~s} \text { at full level }\end{array}$} & \multirow{8}{*}{$\begin{array}{l}\text { Amplitude (10Hz resolution) } \\
20-1000 \mathrm{~Hz}: \quad-1 \mathrm{~dB} /+3 \mathrm{~dB} \\
1000-2000 \mathrm{~Hz}: \quad \pm 3 \mathrm{~dB}\end{array}$} \\
\hline 20 & 0.057 & $\mathrm{~g}^{2} / \mathrm{Hz}$ & & \\
\hline $20-153$ & 0 & $\mathrm{~dB} /$ oct & & \\
\hline 153 & 0.057 & $\mathrm{~g}^{2} / \mathrm{Hz}$ & & \\
\hline $153-190$ & 7.67 & $\mathrm{~dB} /$ oct & & \\
\hline 190 & 0.099 & $\mathrm{~g}^{2} / \mathrm{Hz}$ & & \\
\hline $190-250$ & 0 & $\mathrm{~dB} /$ oct & & \\
\hline 250 & 0.099 & $\mathrm{~g}^{2} / \mathrm{Hz}$ & & \\
\hline $250-750$ & -1.61 & $\mathrm{~dB} /$ oct & & \multirow[t]{5}{*}{ RMS acc.: $\quad \pm 10 \%$} \\
\hline 750 & 0.055 & $\mathrm{~g}^{2} / \mathrm{Hz}$ & & \\
\hline $750-2000$ & -3.43 & $\mathrm{~dB} /$ oct & & \\
\hline 2000 & 0.018 & $\mathrm{~g}^{2} / \mathrm{Hz}$ & & \\
\hline RMS acc. & 9.47 & $\mathrm{~g}$ & & \\
\hline \multicolumn{5}{|c|}{ Resonance Searches } \\
\hline \multicolumn{3}{|c|}{ Levels } & Duration & Tolerances \\
\hline Frequency $(\mathrm{Hz})$ & Value & Unit & $5-2000 \mathrm{~Hz}$ sweep & \multirow{2}{*}{ Same as Sine } \\
\hline $5-2000$ & 0.5 & $\mathrm{~g}$ & $\approx 260$ s per axis & \\
\hline
\end{tabular}

can be used as comparison pre/post vibration to identify any shift in resonance amplitude or frequency revealing structural changes, including non visible damage, which may have occurred during the test. Visual inspections of the instrument and the screw alignment marks were performed after each sine and random vibration. A functional check was initially scheduled to be performed after completion of the vibration testing, however this was later amended to be performed after completing each axis vibration, to verify that the payload survived without sustaining damage. The functional test involved a basic health check of the instrument which ensured all SiPM channels were still functional; any missing channels would suggest damage to either the SiPM array, or the sensitive wire bonded connections on the CoB SIPHRA. Once the health check was successfully completed, a spectrum was generated using the $2 \%$ thoriated welding rods which allowed a rough gauge of the instrument performance; any significant variation in resolution or gain might indicate crystal decoupling as some form of light (signal) loss in the transmission between the scintillator and the SiPM array. The test was considered passed once GMOD successfully produced a spectrum with distinguishable lines from the thoriated welding rods.

To pass the vibration test campaign, a series of pass/fail criteria were defined. Those most relevant are listed below. The vibration test for each axis would be passed if:

1. After comparison of the resonance search before and after each axis vibration, there is less than $5 \%$ shift in frequency, and less than $20 \%$ shift in amplitude, for modes with an effective mass greater than $10 \%$

2. Visual inspections of GMOD find no damage to the GMOD detector or motherboard and no loosening of mounting screws is observed

3. GMOD successfully produces a $\gamma$-ray spectrum with distinguishable spectral lines

Figure 6 shows the pre- and post-resonance search plots for the $\mathrm{X}$-axis vibration. Each column represents 


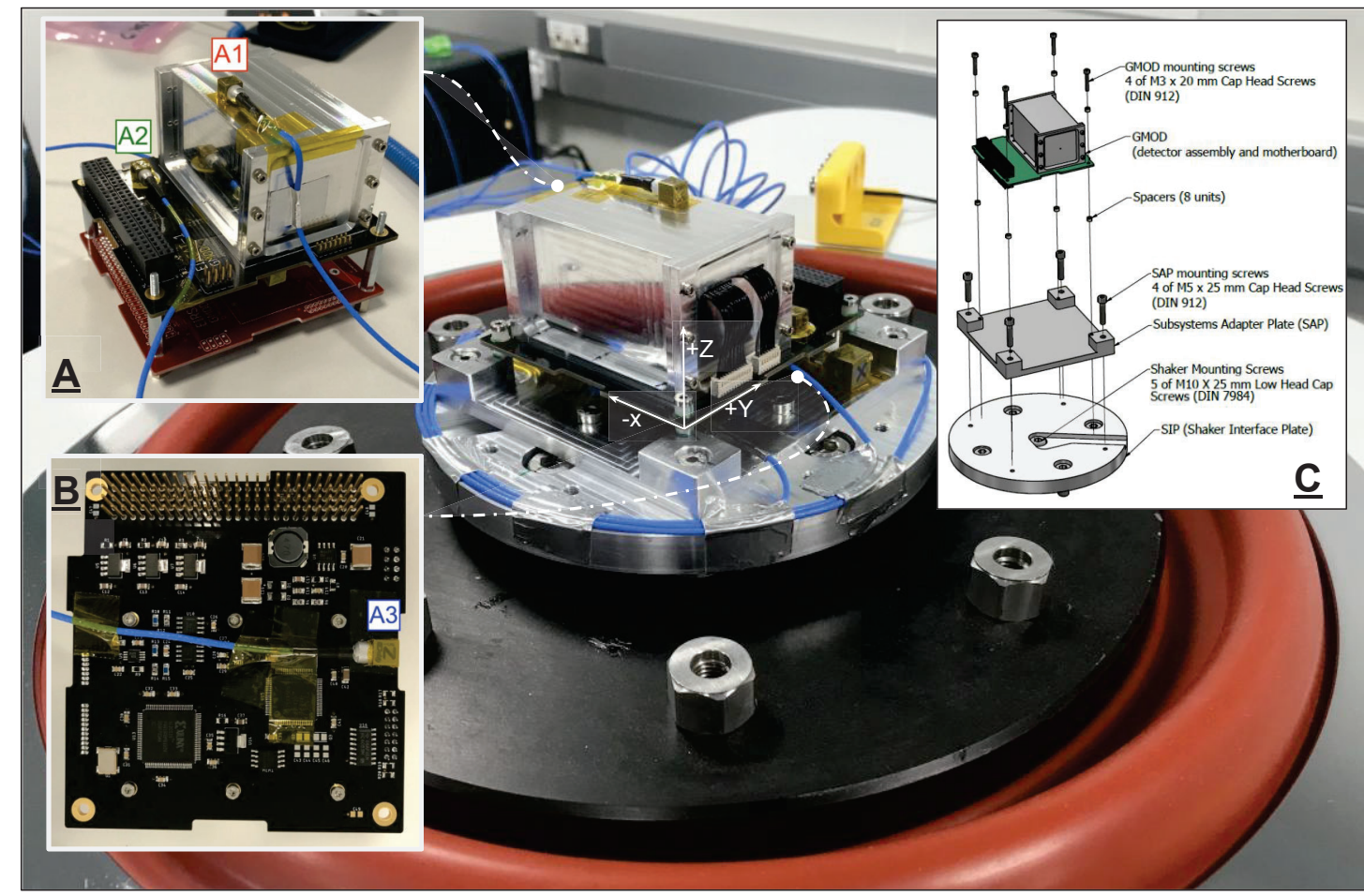

Figure 5: GMOD mounted on the shaker for Z-axis vibration. One of the SIP control accelerometers is visible just beneath the MB PCB. A) Location of the accelerometers A1 and A2. B) Location of accelerometer A3 on the underside of the MB PCB. C) The mounting and orientation of GMOD on the SAP and interface to the shaker using the SIP.

resonance data from a single accelerometer (A1, A2, A3) mounted on GMOD, with each row showing acceleration measurements in the X, Y and Z-axis directions with respect to the CRF. Finally, as specified by the legend, each cell is populated by resonance search profiles for pre-sine, post-sine and post-random (RS1, RS2, RS3) tests, relative to the given accelerometer and axis.

While all other tests passed the pass/fail criteria, the $\mathrm{X}$-axis random vibration failed due to a percentage peak shift greater than the predefined limits in amplitude and frequency. The $\mathrm{X}$-axis was the first axis to undergo vibration testing, starting with a pre-sine resonance search, followed by the sine vibration and post-sine resonance search. Sine vibration was completed successfully, seen in Figure 6 as the RS1 (blue) and RS2 (red) profiles overlap nearly perfectly with little shift in frequency or amplitude. Following this, a visual inspection was performed with no noticeable change to the instrument or its mounting on the shaker table. Random vibration and post-random resonance searches were then conducted. The post-random resonance search profile, RS3 (grey), shows a clear shift in amplitude and frequency after random vibration, as seen in Figure 6. For example, a frequency shift of $11.5 \%$ was observed for the lowest mode, which violates the first pass/fail criteria stated above. Resonance frequency shifts based on the most prominent resonance peaks for both sine and random vibration tests in the $\mathrm{X}$-axis are shown in Table 3 and may be compared to the resonance profiles in Figure 6 .

Table 3: The percentage frequency and amplitude shifts recorded for resonance searches pre/post sine vibration and pre/post random vibration for all accelerometer axes during X-Axis vibration. Any entries highlighted violated the pass/fail criteria. These shifts have been measured from the most prominent resonance peak in both sine and random vibration tests. All other axes passed with shifts less than the predefined limits.

\begin{tabular}{lcccccccccc}
\hline \hline X-Axis Vibration & Profiles & A1X & A1Y & A1Z & A2X & A2Y & A2Z & A3X & A3Y & A3Z \\
\hline Sine \% Freq. Shift & (RS1 $\rightarrow$ RS2) & 1.0 & 0.7 & 1.2 & 1.0 & 0.9 & 0.9 & 2.1 & 1.2 & 0.9 \\
Sine \% Amp. Shift & (RS1 $\rightarrow$ RS2) & 4.3 & 2.7 & 2.4 & 2.7 & 4.6 & 4.8 & 0.4 & 11.3 & 4.4 \\
Random \% Freq. Shift & (RS2 $\rightarrow$ RS3) & 11.3 & 4.8 & 4.2 & 5.0 & 11.5 & 11.5 & 5.0 & 3.9 & 6.8 \\
Random \% Amp. Shift & (RS2 $\rightarrow$ RS3) & 17.0 & 33.9 & 11.5 & 1.0 & 8.2 & 20.8 & 0.8 & 26.8 & 1.7 \\
\hline \hline
\end{tabular}



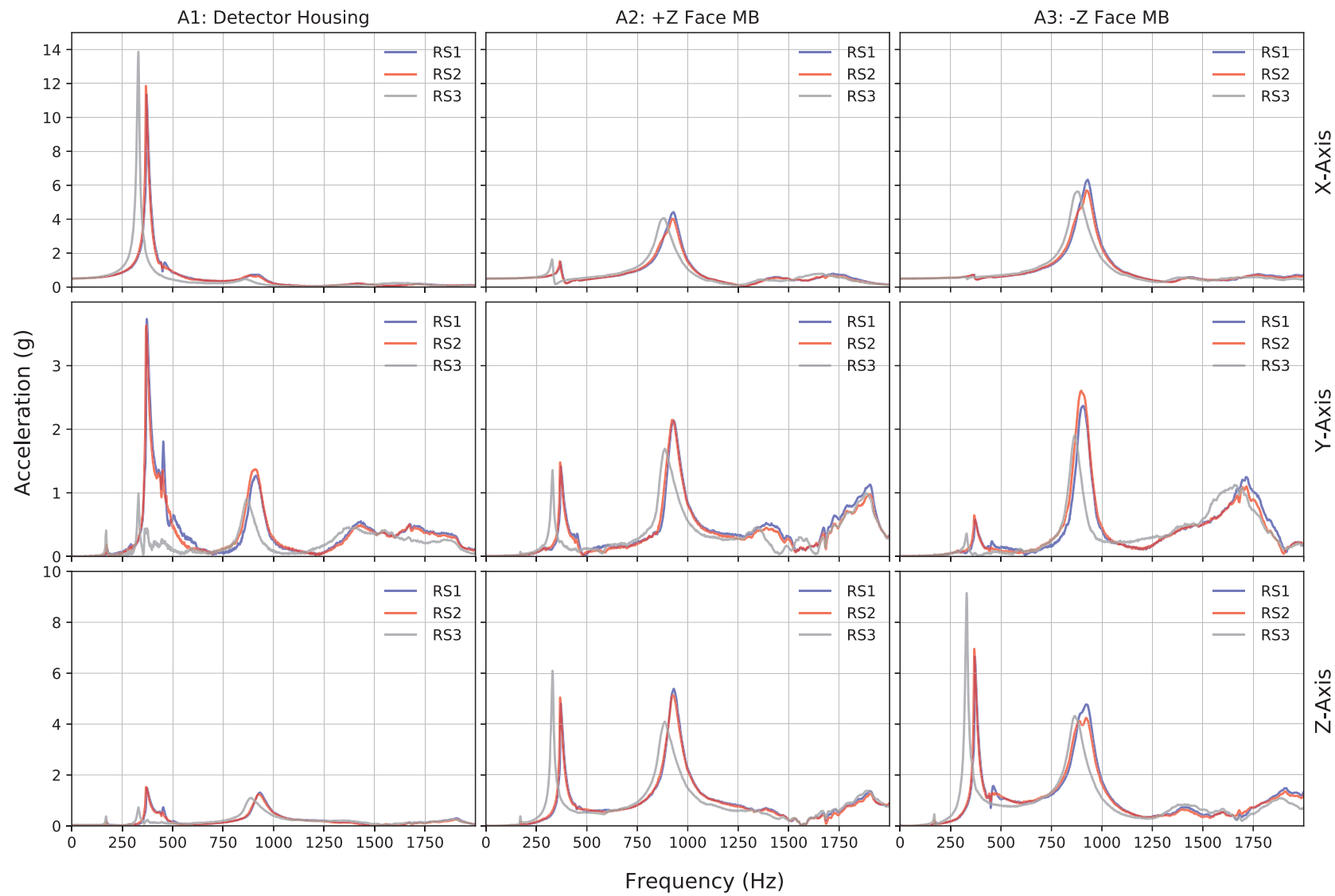

Figure 6: $\mathrm{X}, \mathrm{Y}$ and $\mathrm{Z}$-axis direction resonances (rows) measured during the $\mathrm{X}$-axis vibration testing for each accelerometer (columns) placed on GMOD. Pre/post sine (RS1-blue and RS2-red) and post-random (RS3-grey) resonance searches profiles are shown in each cell whose percentage shifts may be referenced in Table 3.

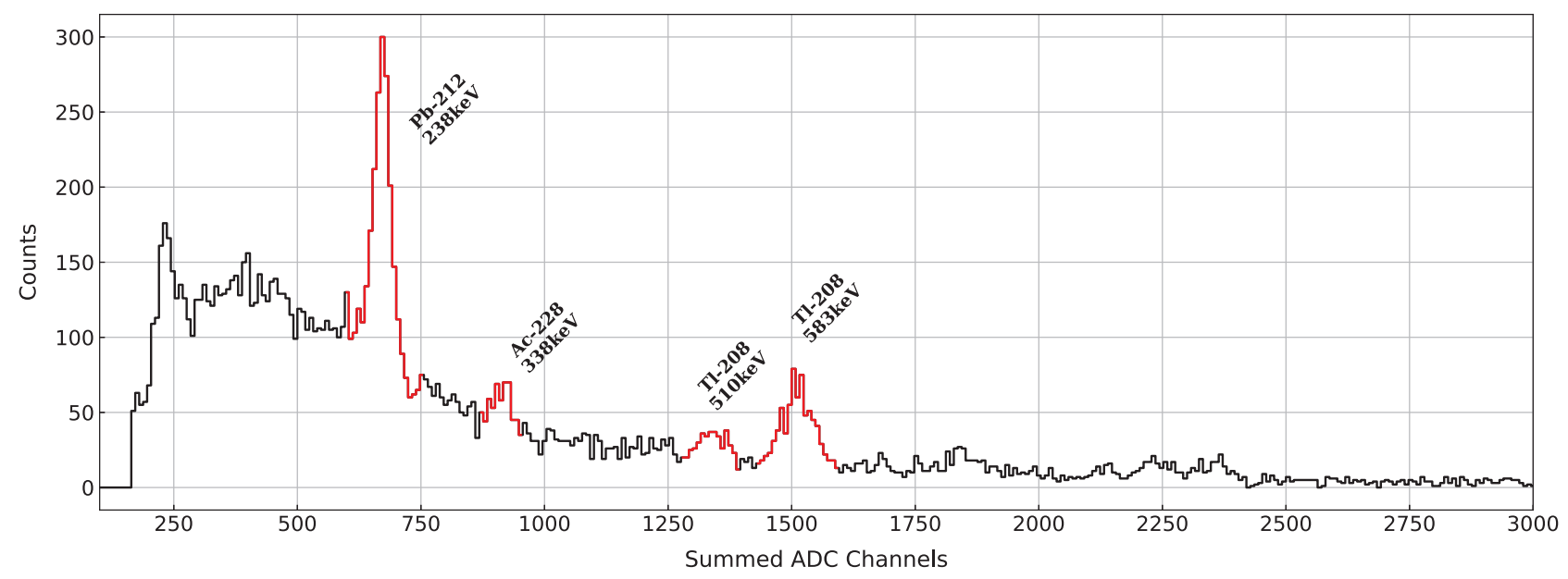

Figure 7: Spectrum generated by GMOD using the thoriated welding rods post $\mathrm{X}$-axis vibration indicating no loss in performance. The expected lines (as referenced in Figure 4) are observable and have been highlighted. The low number of counts is a result of the short integration time being $\sim 10$ minutes in duration. The spectrum has been re-binned from single bins (ADC channels) to 8 channel wide bins to help distinguish the peaks.

A visual inspection of the GMOD MB and detector assembly exterior followed, with particular attention paid to PTFE spacers supporting the MB for any shifting or rotation about their centres which may have occurred. 
No visual changes were noticed and none of the marked spacers indicated any such movement. Due to this shift in frequency a functional test was performed after X-axis vibration. GMOD successfully produced a gamma-ray spectrum with evident spectral lines for the ${ }^{232} \mathrm{Th}$ source during the functional test, as shown in Figure 7 . As GMOD was fully functional, it was deemed appropriate to continue with the Y-axis and Z-axis vibration tests. In the original test procedure, functional testing was foreseen only at the end of the vibration test campaign. After the $\mathrm{X}$-axis anomaly was identified the procedure was modified so that a visual inspection and reduced functional test was carried out after vibration of each axis to monitor the functionality of GMOD throughout the campaign.

Between axes vibration, GMOD was dismounted from the SAP. Between the Y-axis and Z-axis set up, an opportunity to inspect the underside of the MB became available; there was no indication of damage found. After vibration in the remaining axes, all pass/fail criteria were passed successfully, no anomalies were detected and GMOD was proven to be fully functional as a $\gamma$-ray spectrum with clear spectral lines was produced in the functional check. GMOD was then removed from the shaker table and disassembled for the mounting of thermocouple sensors for the TVAC testing. This gave a valuable opportunity to visually inspect the electronics assembly hardware post vibration testing to locate any internal structural changes. Each component in the electronics assembly was carefully disassembled, photographed and cleaned using IPA and lint free wipes. No damage was observed. The following are the potential causes of the frequency shift observed during the $\mathrm{X}$-axis vibration which were derived after root-cause analysis, ordered from most to least likely.

1. Movement of GMOD relative to the test fixture - Four M3 bolts secured GMOD to the SAP and SIP plates that were attached to the shaker table. As with possible cause 4, there is a clearance in these mounting holes which could result in a frequency shift. This could not occur at system level as the X-Y constraints in the satellite stack are much tighter.

2. Relief of stresses introduced during assembly - The components of the detector assembly are tightly packed. During insertion of the components, stresses could be introduced that are then relieved as the surface of the spacers shift in the enclosure during vibration. There are no negative effects to GMOD in this scenario.

3. Movement of internal components - Movement of the scintillator could cause additional compression of the silicone optical pad. Compression would improve optical coupling but the effect would likely be undetected as it would be insignificant.

4. Movement of the Detector Assembly relative to the GMOD Motherboard - There is a small amount of clearance in the holes where M2 bolts are used to secure the detector assembly to the MB. A shift of \pm 0.1 mm could explain the frequency shift observed. It should be noted that these bolts were secured using a thread-locking fluid and showed no sign of loosening.

None of these proposed causes pose any issues for GMOD should they occur during the mission. The vibration test campaign was completed successfully and provided confidence in the mechanical design of GMOD. A NCR was issued for the $\mathrm{X}$-axis shift, and the environmental testing continued as planned.

\subsection{Thermal-Vacuum Testing}

\subsubsection{Pre-Environmental Bake-Out}

Before being shipped to the CSF, the finished GMOD EQM components were baked out under vacuum to prevent the outgassing of contaminants within the CSF TVAC. During thermal-vacuum testing, neither a Residual Gas Analyzer nor a Thermoelectric Quartz Crystal Microbalance were used to monitor outgassing, however a cold trap was used to collect any contaminant which may not have already been outgassed during bakeout. Additionally all other equipment (harnessing, adapters etc.) to be used within the components were verified to be vacuum compatible ${ }^{42}$ before transport.

\subsubsection{Modelling and Simulations}

Simulations were used to determine the expected temperature profile of the satellite while in orbit. A thermal analysis of the spacecraft was performed using the C\&RTech package, Thermal Desktop*, an AutoCAD plugin for general thermal response modelling. The spacecraft model was simplified into a set of geometric bodies with

\footnotetext{
*URL: https://www.crtech.com/products/thermal-desktop
} 
curved surfaces approximated by polygonal structures of similar cross-sectional area. This was done to accelerate the computation time. Simplifications include rounded corners being squared, holes being removed from PCBs, the SiPMs being substituted by silicon wafers and bolts being removed. For other simple volume components (like resistors, capacitors etc), these were assimilated into a single lump mass across the PCB surface. The MSP430 and CoolRunnerII CPLD were retained for their thermal contribution as their profile would vary for operational and non-operational modes. The $\mathrm{CeBr}_{3}$ crystal was modelled as a single block of Cerium for a conservative estimate of the thermal properties, as at the time this model was developed $\mathrm{CeBr}_{3}$ had only just began to be used for scintillation and few sources on the thermal properties were in existence. It was anticipated that the crystal would be heavily insulated due to being wrapped in a number of layers of teflon (as discussed in Section 2) and hermetically encased within its own aluminium housing. The satellite model was simulated in an ISS-like LEO with an initial temperature of $20^{\circ} \mathrm{C}$ over 10 orbits to allow the temperature to stabilise and oscillate about a realistic temperature mean. Sources of radiation within the simulated environment include the Sun, Earth albedo and Infrared (IR) re-emission by the planet. A number of mission scenarios were investigated including operational and non-operational modes during nominal, cold, and solstice* cases. The results provide an in orbit minimum and maximum temperature range for the SiPM array, CPLD and MSP430. It also verified the high thermal insulation of the scintillation crystal with respect to the rest of the instrument.

Table 4: Results of the thermal simulations of GMOD for operational \& non-operational modes under a variety of conditions, with a $10^{\circ} \mathrm{C}$ simulation uncertainty applied (i.e. $10^{\circ} \mathrm{C}$ added to the predicted maximum, subtracted from the predicted minimum). Highlights indicate temperature extremes experienced across all scenarios.

\begin{tabular}{lcccccc}
\hline \hline & \multicolumn{2}{c}{ Nominal } & \multicolumn{2}{c}{ Cold } & \multicolumn{2}{c}{ Solstice } \\
\hline \hline Operational & Min & Max & Min & Max & Min & Max \\
\hline \hline SiPM & $+10^{\circ} \mathrm{C}$ & $+44.1^{\circ} \mathrm{C}$ & $+10^{\circ} \mathrm{C}$ & $+42.3^{\circ} \mathrm{C}$ & $+10^{\circ} \mathrm{C}$ & $+64.6^{\circ} \mathrm{C}$ \\
CPLD & $-5.1^{\circ} \mathrm{C}$ & $+37.8^{\circ} \mathrm{C}$ & $-8.8^{\circ} \mathrm{C}$ & $+35.2^{\circ} \mathrm{C}$ & $+10^{\circ} \mathrm{C}$ & $+49.7^{\circ} \mathrm{C}$ \\
MSP430 & $-5.1^{\circ} \mathrm{C}$ & $+37.8^{\circ} \mathrm{C}$ & $-8.8^{\circ} \mathrm{C}$ & $+35.3^{\circ} \mathrm{C}$ & $+10^{\circ} \mathrm{C}$ & $+49.7^{\circ} \mathrm{C}$ \\
\hline \hline Non-Operational & Min & Max & Min & Max & Min & Max \\
\hline \hline SiPM & $-9.2^{\circ} \mathrm{C}$ & $+30.9^{\circ} \mathrm{C}$ & $-13.6^{\circ} \mathrm{C}$ & $+30.3^{\circ} \mathrm{C}$ & $+7.6^{\circ} \mathrm{C}$ & $+30.0^{\circ} \mathrm{C}$ \\
CPLD & $-16.8^{\circ} \mathrm{C}$ & $+34.9^{\circ} \mathrm{C}$ & $-20.9^{\circ} \mathrm{C}$ & $+32.3^{\circ} \mathrm{C}$ & $+6.6^{\circ} \mathrm{C}$ & $+30.4^{\circ} \mathrm{C}$ \\
MSP430 & $-16.8^{\circ} \mathrm{C}$ & $+34.9^{\circ} \mathrm{C}$ & $-20.9^{\circ} \mathrm{C}$ & $+32.3^{\circ} \mathrm{C}$ & $+6.6^{\circ} \mathrm{C}$ & $+30.4^{\circ} \mathrm{C}$ \\
\hline \hline
\end{tabular}

\subsubsection{Test Levels and Set-Up}

Through simulation, the maximum and minimum temperature extremes experienced while in orbit was found to be $+64.6^{\circ} \mathrm{C}$ and $-20.9^{\circ} \mathrm{C}$ respectively. For the purposes of qualification testing, an additional $\pm 10^{\circ} \mathrm{C}$ qualification margin ${ }^{31,32}$ was added to these temperatures. The minimum qualification temperature was therefore defined as $-31^{\circ} \mathrm{C}$ while the maximum was defined as $+75^{\circ} \mathrm{C}$. The vacuum chamber would be pumped down to a target of $10^{-6} \mathrm{mBar}$ with thermal cycling permitted once $10^{-4} \mathrm{mBar}$ had been achieved. Once the Temperature Reference Point (TRP) had surpassed the qualification threshold the instrument was then set to dwell at the qualification temperature for a duration of 60 minutes. The TRP is a physical location on the IUT chosen as a simplified representation of the unit temperature ${ }^{43}$ and is used as a reference when determining if and for how long the IUT had surpassed the qualification thresholds. The dwell period is allocated to allow sufficient time for thermal conduction to stabilise throughout the IUT while at the qualification temperature.

Following vibration testing, the disassembled GMOD components were cleaned using IPA and lint free wipes before the PT100 sensor and thermocouples were mounted to the GMOD hardware. These would be used for monitoring the thermal behaviour of the components chosen based on the thermal simulations (section 3.3.2). The simulations suggested that the crystal would be heavily thermally insulated within the detector assembly; for this reason, it was of interest to monitor its behaviour. A type $\mathrm{T}$ thermocouple was installed on the crystal housing while a custom endcap was machined with a channel to allow the thermocouple cable to be routed outside once sealed. The PTFE venting channel was enlarged to allow enough room for the cable cross section to pass

${ }^{*}$ This is a specialised case representing the duration that the spacecraft is held in a sustained exposure to sunlight due to the yearly solstices and the orbit configuration. The orbit parameters were adjusted to best represent these effects. 
through. The spacer and endcap are representational only, with minimal modifications to support the testing. The mounting of the crystal thermocouple is shown in Figure $8 \mathrm{C}$ and D which shows the PTFE spacers around the scintillator and the raised surface caused by the thermocouple which was mounted using aluminium tape. The rest of the type $\mathrm{T}$ thermocouples were mounted onto the MB hardware, one on top of the CoolRunnerII CPLD and the other ontop of the MSP430 microcontroller packages. A type T thermocouple to be used as the TRP was also mounted to the MB PCB on a region in the center of the board void of components or traces (as seen in Figure 8A). This location was chosen such that controlling the temperature at this point would effectively drive the temperature of instrument as a whole and could be used to infer it's mean temperature. This installation was conducted by CSF operators using aluminium tape, while layering a strip of kapton tape atop to attempt to match the emissivity properties of the PCB.

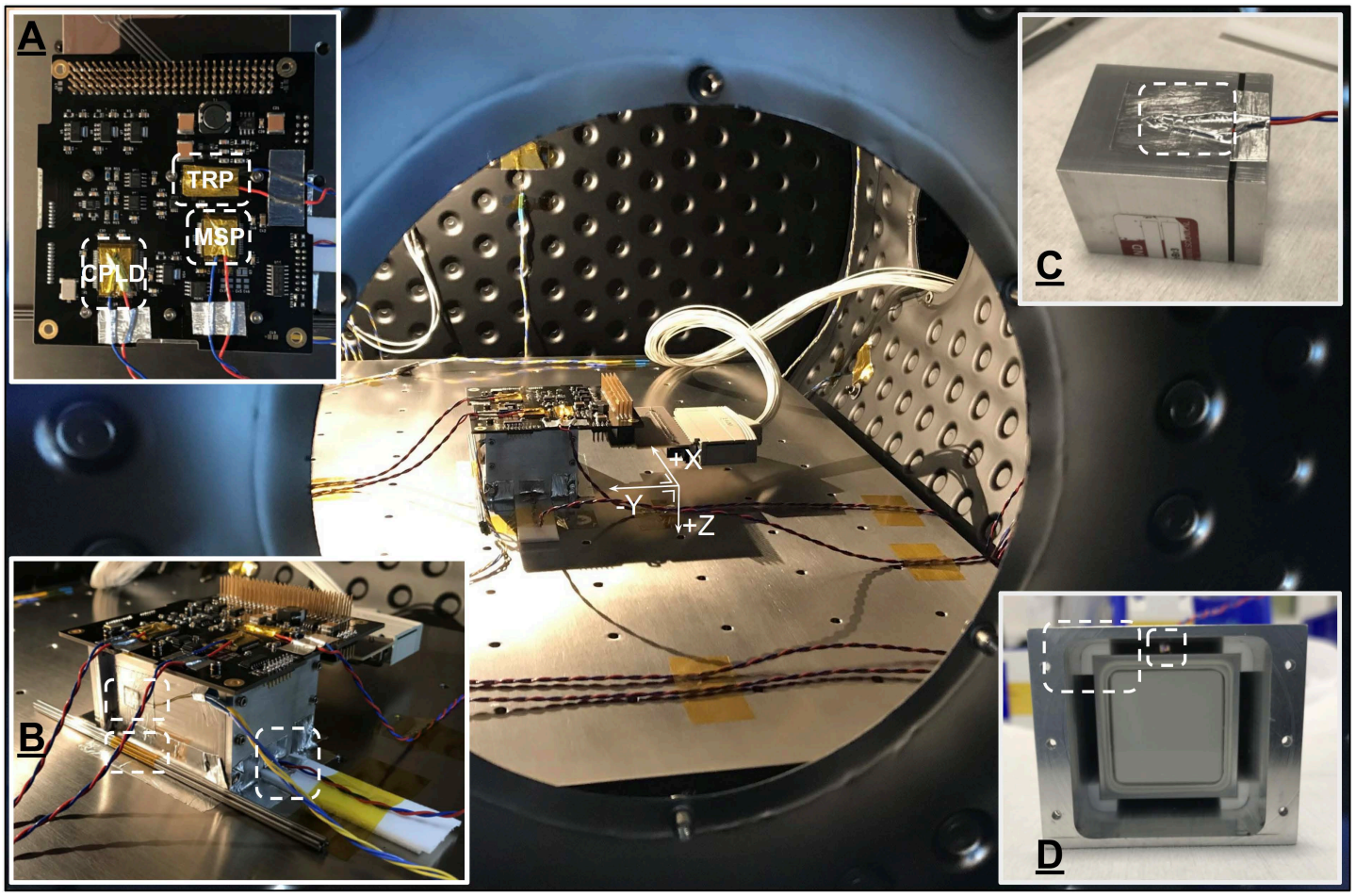

Figure 8: GMOD within the TVAC chamber. A) The placement of thermocouples on the MSP430, CPLD and the TRP with kapton tape overlay. B) The placement of GMOD in the TVAC, the throiated welding rods, PT100 mounting and the crystal thermocouple cable. C) The crystal thermocouple mounted on the crystal aluminium container surface. D) The crystal within the detector assembly housing supported with PTFE spacers.

Table 5: TVAC test levels and durations, with phase overview and durations spent beyond qualification thresholds below. * Note: for cold cycle durations in Time Over $T_{Q}, \mathrm{~T}_{Q M i n}$ is measured as the lower bandwidth threshold of $<-30^{\circ} \mathrm{C}, 1^{\circ} \mathrm{C}$ greater than the predefined $\mathrm{T}_{Q M i n}$.

\begin{tabular}{|c|c|c|c|c|c|c|c|c|c|}
\hline Parameter & \multicolumn{2}{|c|}{ Level } & \multicolumn{7}{|c|}{ Notes } \\
\hline $\mathrm{T}_{Q \operatorname{Max}}$ & \multicolumn{2}{|c|}{$+75^{\circ} \mathrm{C}$} & \multicolumn{7}{|c|}{ Bandwidth $\left[+75^{\circ} \mathrm{C},+78^{\circ} \mathrm{C}\right]$} \\
\hline $\mathrm{T}_{Q M i n}$ & \multicolumn{2}{|c|}{$-31^{\circ} \mathrm{C}$} & \multicolumn{7}{|c|}{ Bandwidth $\left[-30^{\circ} \mathrm{C},-34^{\circ} \mathrm{C}\right]$} \\
\hline Pressure & \multicolumn{2}{|c|}{$10^{-6} \mathrm{mBar}$} & \multicolumn{7}{|c|}{ Cycling permitted beyond $10^{-4} \mathrm{mBar}$} \\
\hline Dwell & \multicolumn{2}{|c|}{$1 \mathrm{hr}$} & \multicolumn{7}{|c|}{ Updated to $\sim 2 \mathrm{hrs}$ after Non-Op } \\
\hline Cycles & \multicolumn{2}{|c|}{4} & \multicolumn{7}{|c|}{1 Non-Op 3 Operational (+ 1 half final $)$} \\
\hline & \multicolumn{2}{|c|}{ Non-Op } & \multicolumn{7}{|c|}{ Operational } \\
\hline Phase & $\mathbf{I}$ & II & III & IV & $\mathbf{V}$ & VI & VII & VIII & IX \\
\hline Range & Hot & Cold & Hot & Cold & Hot & Cold & Hot & Cold & Hot \\
\hline Time Over $\mathrm{T}_{Q}^{*}$ & 1:06:05 & 1:45:40 & 01:40:20 & 01:02:35 & $1: 33: 20$ & $1: 03: 15$ & $1: 35: 55$ & 1:03:45 & $1: 07: 20$ \\
\hline
\end{tabular}


An additional PT100 sensor was mounted on the aluminium housing on the $-\mathrm{Y}$ face (with respect to the $\mathrm{CRF}$ ), kapton tape was not used in this case as the emissivity of the aluminium tape better matched that of the aluminium housing. GMOD was then installed within the $\mathrm{S}^{3}$ TVAC chamber (Figure 8); thermocouple cables were routed through the chamber through a feed through block, while GMOD power and data EGSE were connected using the custom vacuum compatible interface PCB via a D-sub 37 cable. GMOD was inverted within the chamber so the aluminium housing interfaced with the thermal plate, with the addition of aluminium tape to secure good thermal contact. The thoriated welding rods were placed beside the detector assembly along its longest axis as a source of gamma-ray emission for the operational cycle spectral measurements. The PT100 placement, thoriated welding rods and crystal thermocouple sensor cable can be seen in Figure 8B. Externally, the thermocouples were routed to the CSF data acquisition hardware, while the GMOD EGSE connected to the external interface board, providing power, command and data readout via the custom UI, discussed in Section 3.1.

\subsubsection{Thermal-Vacuum Test Operation \& Results}

The TVAC was pumped down from ambient pressure to $10^{-6} \mathrm{mbar}$; after reaching a minimum of $10^{-4} \mathrm{mbar}$, thermal cycling began with a non-operational thermal cycle later followed by three operational* cycles. The TVAC under vacuum would manage these cycles by varying the temperature at a rate of $2^{\circ} \mathrm{C} / \mathrm{min}$ (via the thermal interface plate) from ambient temperature to $+75^{\circ} \mathrm{C}$ with a dwell at that temperature until the instrument reached thermal equilibrium (originally 1 hour duration). The cycle would then proceed to the cold cycle where the thermal plate would drop in temperature below ambient to $-31^{\circ} \mathrm{C}$ with another dwell, as before to ensure thermal equilibrium. A final half cycle (from ambient temperature to hot and returning to ambient) was done to allow the detector to return to ambient from a hot cycle phase to prevent any condensation or residual moisture build up within the chamber after repressurisation and to discourage dendritic ${ }^{44}$ growth on the SIPHRA silicon die. The TVAC thermal and pressure measurements are shown in Figure 9.

To pass the TVAC test campaign, a series of pass/fail criteria were defined. Those most relevant are listed below. The TVAC testing would be passed if:

1. The GMOD instrument completes all functional tests and cycles (operational and non-operational) without sustaining damage due to high or low temperature stresses

2. Detailed visual checks of the GMOD instrument before and after the test campaign show no physical damage to the instruments detector assembly, MB, PCB traces or components (ie. thermal cycling causing cracking, $\mathrm{PCB}$ components functionality impaired, any occurrence which results in the GMOD instrument becoming inoperable)

3. The instrument can perform the experiment and successfully generate a spectrum, during the operational cycles.

During the non-operational cycle, as expected the crystal was found to be very well thermally isolated from the rest of the instrument, with significant lag between the crystal temperature and the other sensor measurements (Figure 9, Phases I \& II). The first, non-operational cycle reached its dwell and qualification criteria without incident, after which GMOD was powered up and a serial connection was established while rising from the nonoperational cold cycle, into the operational phases (Phases III to IX). A successful health check was performed around $0^{\circ} \mathrm{C}$ confirming the instrument had survived the non-operational cycle. GMOD was then commanded to enter operational mode and begin measuring a spectrum leading into the operational cycling stage. The first operational phase (Phase III) demonstrated the crystals extreme thermal insulation; at one point the crystal showed a temperature differential of $>70^{\circ} \mathrm{C}$ between the crystal and the rest of the instrument (referencing the TRP). An unforeseen effect of this was that the large differential allowed the crystal to act as a heat sink, drawing heat through conduction from the rest of the instrument. While the level of thermal insulation of the crystal was predicted, this behaviour was not. This became problematic during the initial hot dwell of Phase III. Just before the TRP reached $+75^{\circ} \mathrm{C}$, the qualification temperature, it was drawn down below this by the crystal's heat sink behaviour, below that required to achieve the predefined qualification level. This is seen in Figure 9 just beneath the $+75^{\circ} \mathrm{C}$ mark in Phase III. The CSF operators attempted to mitigate the effects of this by adapting the

${ }^{*}$ This involves the power-up of the instrument, a health and functionality check followed by continuous spectral integration using the ${ }^{232} \mathrm{Th}$ containing rods as a gamma-ray emission source. 
scripted TVAC control profile resulting in an increased dwell duration from 1 to 2 hours in duration. Despite this, the crystal's behaviour resulted in multiple dwell stabilisation infractions, compounding the matter, it was impossible to manually adjust the thermal plate setpoint for the remaining two cycles (Phases V - VIII) as it would be performed through automation, overnight and without an attending operator. This would cause the TRP to hover just above the cold dwell threshold qualification of $-31^{\circ} \mathrm{C}$ for all operational cycles (Phase IV, VI, VIII) and exceed at some points the upper limit hot dwell threshold of $+78^{\circ} \mathrm{C}$ (Phases III, V, VII). Regardless, GMOD continued to function as expected without any impact or impedance to performance. An NCR detailing the non conformance was produced outlining the thermal behaviour of the crystal, but stating that there was sufficient confidence that the instrument was properly stressed for qualification and that the objectives of the test were met. This is due in part to the close proximity of the cold dwell qualification temperature to that which was actually reached, a difference within just $1^{\circ} \mathrm{C}$. Furthermore, it was deemed that the test levels were far more demanding than those defined in the ECSS standards. Additionally GMOD is yet to undergo system level qualification testing, while integrated with the rest of the EIRSAT-1 payload, which will provide further confidence in the qualification. Upon completion of the fourth and final cycle of the TVAC campaign (Phases VII to VIII), the instrument was returned to ambient from a hot cycle (Phase IX). The instrument was finally removed and disassembled for visual inspection. All thermocouples were removed and components were inspected and photographed. As no observable damage was discovered and GMOD successfully generated a spectrum, all pass/fail criteria were passed successfully. The duration achieved at qualification levels for each phase during the TVAC campaign are shown in Table 5.

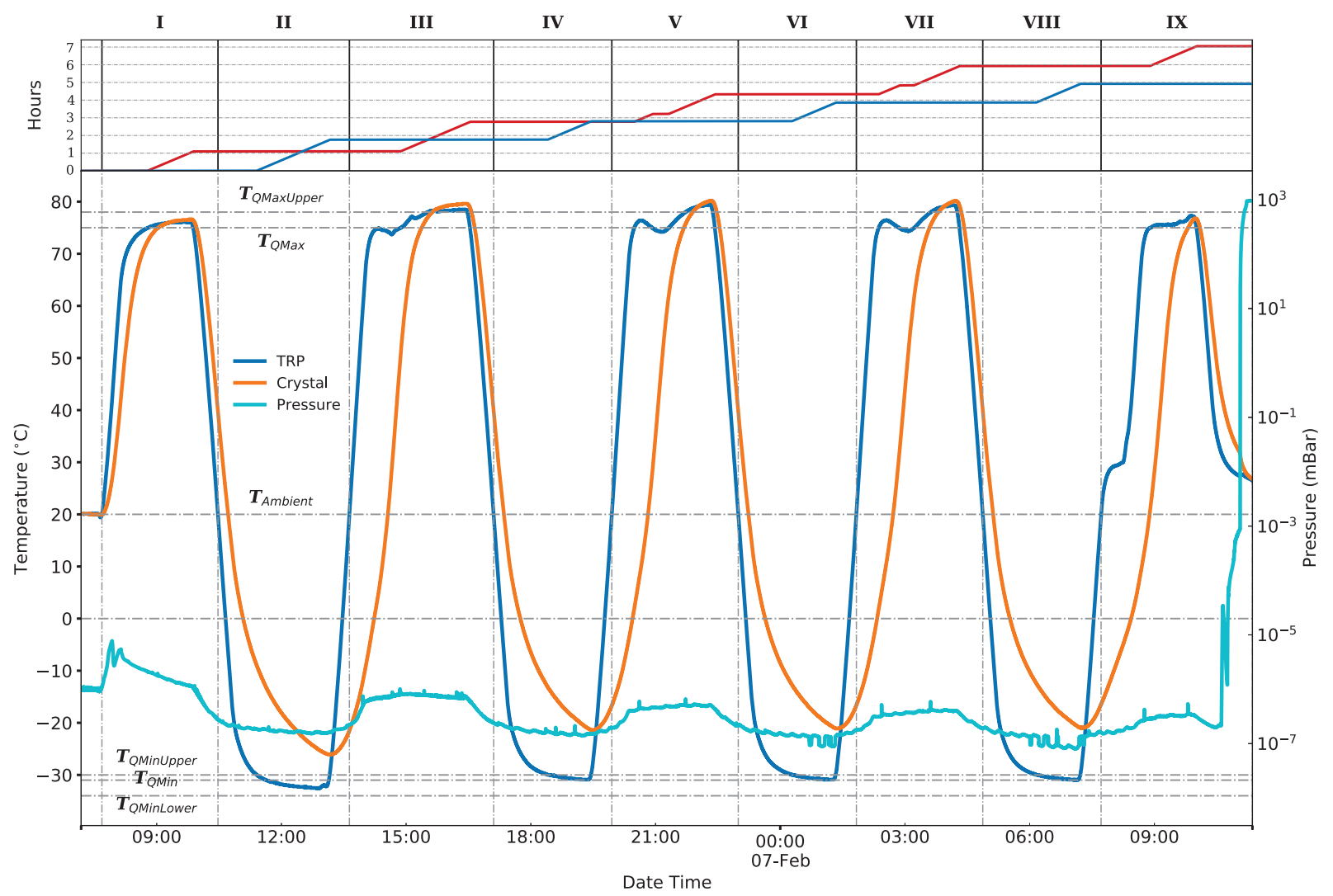

Figure 9: TVAC cycling profile with dwell duration for qualification ranges above in hours. Measurements for the MSP430 and CPLD overlap almost perfectly with the TRP measurements with a maximum difference of $\sim 1.4^{\circ} \mathrm{C}$. For the sake of clarity they have been omitted here. The durations in hours above may be also referenced in Table 5 where $\mathrm{T}_{Q M \text { in }}$ is taken as the lower bandwidth threshold of $-30^{\circ} \mathrm{C}$.

SiPMs operate above the so-called breakdown voltage, in the case of GMOD this is a negatively applied bias voltage. The overvoltage, the excess voltage above breakdown, defines the internal amplification (gain) of 
the SiPM. The breakdown voltage is known to depend on temperature. The temperature dependence of SiPM breakdown voltage and the resulting effects on their characteristics are well known ${ }^{45}$ and were expected to be encountered during the testing. For J-Series $\mathrm{SiPMs},{ }^{46}$ the breakdown voltage is known to vary by $21.5 \mathrm{mV} /{ }^{\circ} \mathrm{C}$. In brief, SiPM gain is defined as the amount of charge released per single photon absorption and is a function of the SiPM overvoltage. Since breakdown voltage is temperature dependent, for a fixed applied bias, any variations in temperature will proportionally vary the overvoltage, and by consequence, the SiPM gain. Thus for a scintillation signal produced by a gamma-ray photon (of a given energy) the amount of charge released by the SiPM array will be either increased or decreased. From a high level perspective, for a fixed applied bias voltage and variable temperature, this will result in a shifting of spectral features and a smearing of any long duration spectral integration when performing pulse height spectroscopy. Another effect is a strong influence of temperature on the SiPM dark current and noise, also functions of temperature. SIPHRA readout of the SiPM array is triggered when the current signal from the detector surpasses a fixed trigger threshold level. Normally this threshold is set sufficiently above the thermally generated dark current signal, such that any fluctuations contributed by SiPM noise do not trigger SIPHRA readout. In the event that the average dark current surpasses the trigger threshold due to the strong influence of temperature, SIPHRA will enter a state where it is constantly triggering on dark current signal at its maximum rate, saturating the readout bandwidth with noise events. This can be seen when SIPHRAs output is dominated by successive triggers with low Analogue to Digital Converter $(\mathrm{ADC})$ value.

While in orbit, GMOD would counter these effects by compensation using temperature reference measurements and adjusting accordingly the bias voltage or SIPHRA configuration to maintain consistent performance. However, due to the GMOD firmware being in an early stage of development, these compensation mechanisms were not implemented for the TVAC testing, thus the thermal effects and their impacts are shown in Figure 10. These effects include temperature induced variations on SiPM gain and dark current fluctuation.
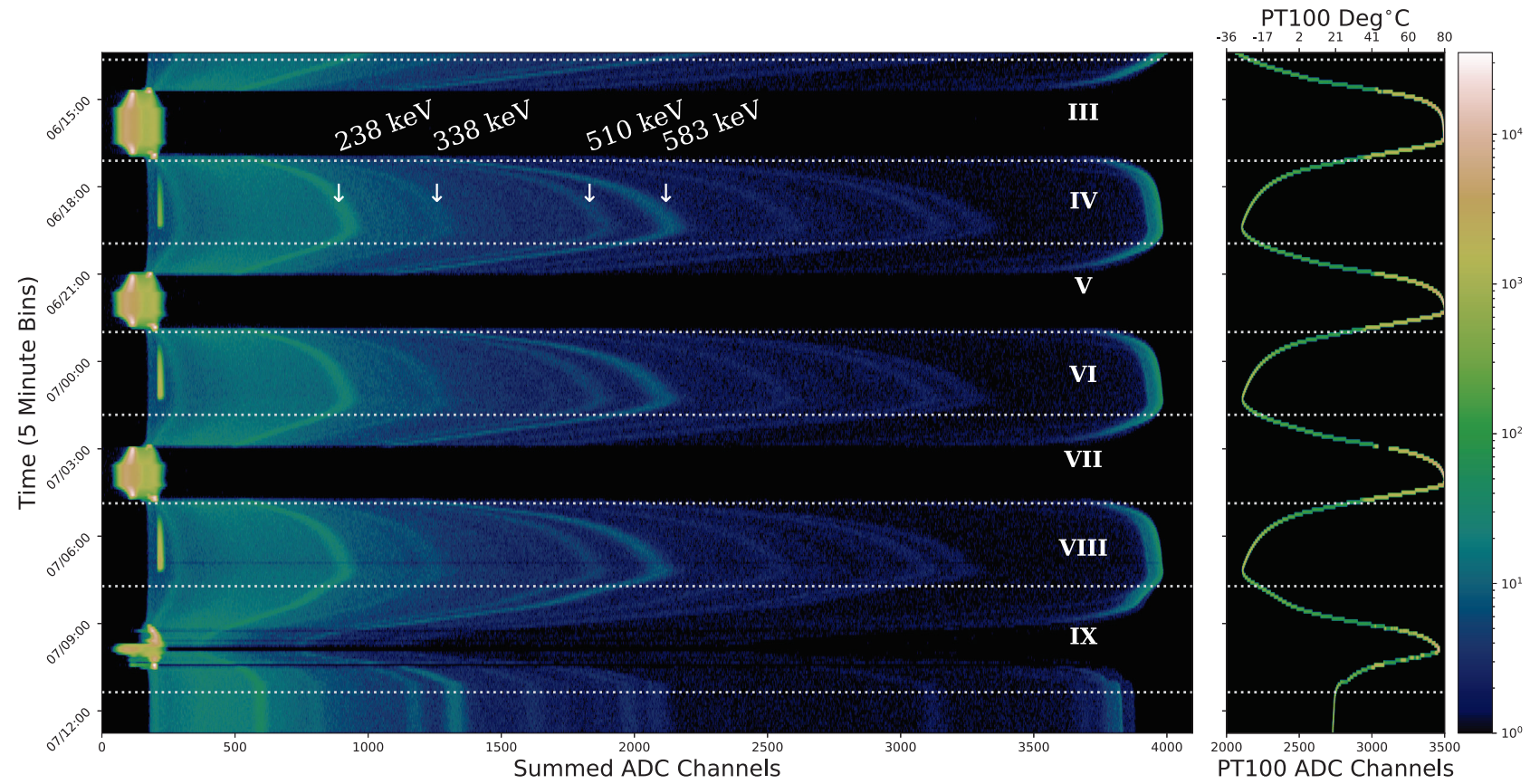

Figure 10: The operational cycle spectral measurements in time resolved format obtained during TVAC cycling. Each slice along the horizontal is an individual spectrum, measured over a given time bin interval. The effects of temperature induced gain variation (as described in Section 3.3.4) are apparent, however the required spectral features remain clearly distinguishable, as seen in Figure 4 \& 7. Each phase of the testing has also be labelled as in Figure 9, with the SiPM PT100 data included with a calibration applied for temperature reference.

To examine the detector performance as a function of temperature without smearing from temperature 
induced gain shifting, the plot is arranged in time resolved format binned into 5 minute intervals (along the y-axis), chronologically from the top down. The shade intensity represents counts per pixel for each ADC bin of spectral data (along the $\mathrm{x}$-axis), in other words, each slice along the horizontal is a individual spectrum at a given time interval, where the most prominent peaks generated by the ${ }^{232} \mathrm{Th}$ source can be distinguished by their colour intensity without being smeared or obscured by dark count saturation. A PT100 is present on the underside of the SiPM array PCB which is digitised and read out by SIPHRA upon trigger (as discussed in Section 2). These measurements are also included for reference with a temperature calibration, where again, the shade intensity represents counts per pixel for each ADC bin of PT100 data. From Figure 10, it can be seen that the prominent spectral features (the $238 \mathrm{keV}$ line in particular) are shifted down in ADC channels with increase in temperature and up in ADC channels with decrease in temperature. However this continuous shifting of the features is broken by voids across all but the lowest ADC channels, during the rise up to and descent from the hot dwell. At these times the SiPM array has reached such a temperature, that the intensity of the dark current has exceeded the fixed trigger threshold, producing a spectrum saturated with noise events. The instrument then returns to normal operation when the temperature has reduced below $\sim 40-50^{\circ} \mathrm{C}$, showing standard readout as the dark current level descends below the trigger threshold. It should be noted, there are a number of ways to mitigate the effects of these behaviours, which unfortunately could not be incorporated into the MSP430 firmware at the time of these tests. This includes bias voltage compensation (to maintain a constant overvoltage) as well as adjustment of the trigger threshold. This final method, was actually demonstrated during the final half cycle (Phase IX) towards the end of the TVAC testing with some success. Figure 10 shows the spectrum during the last hours of the test to be interrupted by saturation only briefly, while the trace of the $232 \mathrm{keV}$ peak is just clear enough to be seen consistently. That said this is impractical and quite difficult to perform, particularly if intended to be automated while in flight. Certainly this may need to be adjusted during the mission lifetime (for example, due to cumulative irradiation effects on the $\mathrm{SiPMs}^{21}$ ), however SIPHRA is capable of subtracting the average current before trigger, thus any increase in dark current may be compensated without need of adjusting the threshold level. In the context of the limitations imposed without this being available for the campaign, the instrument performed as expected and was considered to have passed the predefined pass/fail criteria concluding the TVAC campaign.

\section{POST ENVIRONMENTAL TESTING}

Upon return to Dublin, GMOD was tested one final time to ensure damage had not been sustained during disassembly, reassembly or transport back to University College Dublin (UCD). This was also an opportunity to measure the performance using standard calibration sources, in this case ${ }^{22} \mathrm{Na},{ }^{137} \mathrm{Cs}$ and ${ }^{60} \mathrm{Co}$ which can be more readily used to compare performance as opposed to the ${ }^{232} \mathrm{Th}$ isotope found in the welding rods. This test was carried out informally and is considered supplementary to the suite of tests carried out for the qualification campaign. The same standards for success were maintained, specifically the criteria that the instrument would function as intended and a spectrum could be successfully measured. The generated spectra indicated favourable performance and a return to nominal operations, with a measured resolution of $4.2 \%$ at $662 \mathrm{keV}$.

\section{CONCLUSIONS \& FUTURE DEVELOPMENT}

Following the environmental tests described in this paper and subsequent review by the ESA FYS project team and technical experts, GMOD has successfully completed its qualification, providing confidence in the instrument's design, construction and performance from launch to operations as a payload onboard EIRSAT1. The qualification tested GMOD under environmental scenarios which it would need to survive in order to carry out its mission. This testing stressed the instrument based on qualification margins derived from simulations, ECSS standards and in agreement with FYS coordinators. As such, after successful completion of the environmental campaign, the EQM was deemed to have been qualified for launch. Regarding the performance of GMOD, the instrument survived the environmental testing and was demonstrated to return to normal operating standards after returning to ambient conditions. This was true for the vibration campaign, which saw a nonconformance and shift after X-axis vibration, and the TVAC campaign which saw the unexpected heat sink behaviour of the $\mathrm{CeBr}_{3}$ scintillator. A number of possible sources of the $\mathrm{X}$-axis vibration shift have been presented in order of most to least likely, none of which pose an issue for the instrument operation or require preventative 
actions or corrections. Similarly, no corrective actions were deemed necessary regarding the unexpected thermal behaviour of the crystal during the thermal-vacuum testing. The TVAC test in particular tested the instrument's performance far beyond the nominal cycling while in orbit, showing that the instrument could reliably measure gamma-ray spectra up to $\sim 40-50^{\circ} \mathrm{C}$ before noise saturation, covering the expected temperature range for normal in-orbit operation. Furthermore, methods to mitigate the effects of temperature dependence have been outlined and will be used while in flight to stabilise the instrument performance. Recently the EIRSAT-1 qualification model has been completed with the GMOD EQM integrated into the spacecraft stack in preparation for mission testing ${ }^{47}$ and system level environmental qualification. This will help to provide further confidence in the integrity of the design of GMOD, as already evident from the subsystem level environmental testing which is presented here. Following this will be the completion of the Flight Model, acceptance and delivery to ESA, where it will be prepared for launch into orbit and contribute to multi-messenger astronomy with the GRB community.

\section{ACKNOWLEDGMENTS}

The EIRSAT-1 project is carried out with the support of ESA's Education Office under the Fly Your Satellite! 2 programme. This study was supported by The European Space Agency's Science Programme under contract 4000104771/11/NL/CBi. JM, AU, DM, and SMB acknowledge support from Science Foundation Ireland (SFI) under grant number 17/CDA/4723. LH acknowledges support from SFI under grant 19/FFP/6777. DM, RD, JT, COT, MD and LS acknowledge support from the Irish Research Council (IRC) under grants GOIPG/2014/453, GOIPG/2019/2033, GOIPG/2014/684, GOIPG/2017/1031, GOIP/2018/2564 and GOIPG/2017/1525 respectively. JE and JR acknowledge scholarships from the UCD School of Physics and FM acknowledges support from the School of Computer Science. We acknowledge all students who have contributed to EIRSAT-1. The authors would like to thank the ESA Fly Your Satellite! coordinators, operators and personnel for their guidance and assistance throughout this qualification campaign. 


\section{REFERENCES}

[1] Vedrenne, G., Atteia, J.-L., and Cominsky, L., [Gamma-Ray Bursts: The Brightest Explosions in the Universe], Springer (2009).

[2] Zhang, B., [The Physics of Gamma-Ray Bursts], Cambridge University Press (2018).

[3] Narayana Bhat, P. and et al., "The Third Fermi GBM Gamma-Ray Burst Catalog: The First Six Years," https://iopscience. iop. org/journal/2041-8205 223, 28 (Apr 2016).

[4] MacFadyen, A. I. and Woosley, S. E., "Collapsars: Gamma-Ray Bursts and Explosions in "Failed Supernovae"," https: // iopscience. iop. org/ journal/2041-8205 524, 262-289 (Oct. 1999).

[5] Blinnikov, S. I., Novikov, I. D., Perevodchikova, T. V., and Polnarev, A. G., "Exploding Neutron Stars in Close Binaries," Soviet Astronomy Letters 10, 177-179 (Apr. 1984).

[6] Paczynski, B., "Gamma-ray bursters at cosmological distances," https://iopscience. iop.org/ journal/2041-8205 308, L43-L46 (Sept. 1986).

[7] Abbott, e. a., "Gravitational Waves and Gamma-Rays from a Binary Neutron Star Merger: GW170817 and GRB 170817A," https: // iopscience. iop. org/ journal/2041-8205 848, L13 (Oct. 2017).

[8] Harry, G. M. and Collaboration, L., "Advanced LIGO: the next generation of gravitational wave detectors," Classical and Quantum Gravity 27, 084006 (Apr. 2010).

[9] Collaboration, L., "Advanced LIGO," Classical and Quantum Gravity 32, 074001 (Apr. 2015).

[10] Goldstein, A., Veres, P., Burns, E., Briggs, M. S., et al., "An Ordinary Short Gamma-Ray Burst with Extraordinary Implications: Fermi-GBM Detection of GRB 170817A," https://iopscience. iop. org/ journal/2041-8205 848, L14 (Oct 2017).

[11] Gehrels, N. et al., "The Swift Gamma-Ray Burst Mission," The Astrophysical Journal 611, 1005-1020 (Aug. 2004).

[12] Meegan, C. et al., "The Fermi Gamma-ray Burst Monitor," The Astrophysical Journal 702, 791-804 (Sept. 2009).

[13] Atwood, W. B. and et al., "The Large Area Telescope on the Fermi Gamma-Ray Space Telescope Mission," The Astrophysical Journal 697, 1071-1102 (may 2009).

[14] Winkler, C. et al., "The INTEGRAL mission," Astronomy and Astrophysics 411, L1-L6 (Nov. 2003).

[15] Perkins, e. a., "BurstCube: A CubeSat for Gravitational Wave Counterparts," in [American Astronomical Society Meeting Abstracts \#231], American Astronomical Society Meeting Abstracts 231, 361.14 (Jan. 2018).

[16] D. Murphy, J. Thompson and others , "EIRSAT-1: The Educational Irish Research Satellite," Proceedings on the 2nd Symposium on Space Educational Activities (Apr 2018).

[17] The European Space Agency, "ESA - Fly Your Satellite!."

[18] Ulyanov, A., Morris, O., Hanlon, L., McBreen, S., Foley, S., Roberts, O. J., Tobin, I., Murphy, D., Wade, C., Nelms, N., Shortt, B., Slavicek, T., Granja, C., and Solar, M., "Performance of a Monolithic LaBr 3 :Ce Crystal Coupled to an Array of Silicon Photomultipliers," Nuclear Instruments and Methods in Physics Research A 810, 107-119 (Feb 2016).

[19] Ulyanov, A., Morris, O., Roberts, O. J., Tobin, I., Hanlon, L., McBreen, S., Murphy, D., Nelms, N., and Shortt, B., "Localisation of Gamma-Ray Interaction Points in Thick Monolithic $\mathrm{CeBr}_{3}$ and $\mathrm{LaBr}_{3}: \mathrm{Ce}$ Scintillators," Nuclear Instruments and Methods in Physics Research A 844, 81-89 (Feb 2017).

[20] Ulyanov, A., Murphy, D., Fredriksen, A., Ackermann, J., Meier, D., Nelms, N., Shortt, B., McBreen, S., and Hanlon, L., "Using the SIPHRA ASIC with an SiPM array and scintillators for gamma spectroscopy," in [2017 IEEE Nuclear Science Symposium and Medical Imaging Conference (NSS/MIC)], 1-3 (2017).

[21] Ulyanov, A., Murphy, D., Mangan, J., Gupta, V., Hajdas, W., de Faoite, D., Shortt, B., Hanlon, L., and McBreen, S., "Radiation Damage Study of SensL J-series Silicon Photomultipliers Using 101.4 MeV Protons," Nuclear Instruments and Methods in Physics Research Section A: Accelerators, Spectrometers, Detectors and Associated Equipment 976, 164203 (2020). https://doi.org/10.1016/j.nima.2020.164203.

[22] Renker, D. and Lorenz, E., "Advances in Solid State Photon Detectors," Journal of Instrumentation 4, P04004-P04004 (apr 2009).

[23] G A Quarati, F et al., "Scintillation and Detection Characteristics of High-Sensitivity CeBr3 Gamma-Ray Spectrometers," 729, 596 (10 2013). 
[24] Meier, D., Ackermann, J., Olsen, A., Kristian, H., Berge, H., Hasanbegovic, A., Altan, A., Azman, S., Najafiuchevler, B., Talebi, J., Påhlsson, P., Steenari, D., Fredriksen, A., Øya, P., Johansen, M., Gheorghe, C., Stein, T., and Maehlum, G., "SIPHRA 16-Channel Silicon Photomultiplier Readout ASIC," (06 2016).

[25] Doyle, M., Gloster, A., O'Toole, C., Mangan, J., Dunwoody, R., Thompson, J., and Sherwin, D., "Flight Software Development for the EIRSAT-1 mission," Proceedings of the 3rd Symposium on Space Educational Activities (2020).

[26] Bagagli, R. and et al., "Environmental Tests of the Flight GLAST LAT Tracker Towers," Nuclear Instruments and Methods in Physics Research Section A: Accelerators, Spectrometers, Detectors and Associated Equipment 584, 358-373 (01 2008).

[27] Monteiro, J. P., Rocha, R. M., Silva, A., Afonso, R., and Ramos, N., "Integration and Verification Approach of ISTSat-1 CubeSat," Aerospace 6(12) (2019).

[28] The European Space Agency for the members of ECSS, [ECSS-M-ST-10C - Space project management: Project planning and implementation], The European Space Agency (ESA) (2009).

[29] The European Space Agency for the members of ECSS, [ECSS-E-HB-10-02A - Verification guidelines], The European Space Agency (ESA) (2010).

[30] Walsh, Sarah and et al., "Assembly Integration and Verification Activities for a 2U CubeSat EIRSAT-1," (Apr 2020).

[31] The European Space Agency for the members of ECSS, [ECSS-E-ST-10-03C - Space engineering: Testing], The European Space Agency (ESA) (2012).

[32] The European Space Agency for the members of ECSS, [ECSS-E-ST-10-03A - Space engineering: Testing], The European Space Agency (ESA) (2002).

[33] The European Space Agency for the members of ECSS, [ECSS-Q-ST-10-09C - Space product assurance: Nonconformance control system], The European Space Agency (ESA) (2018).

[34] Laboratory, I. N. E. . E., [Gamma-Ray Spectrum Catalogue: Ge(Li) and Si(Li) Detector Spectra], Idaho National Engineering \& Environmental Laboratory (1999 (Original 1974)).

[35] ISO - International Organization for Standardization, [Arc Welding and Cutting - Tungsten Electrode Classification (ISO 6848: 2004)], ISO (2004).

[36] UK Ministry of Defense and Defense Safety Authority, [Management of radiation protection in defence: part 2 guidance (JSP 392): Chapter 29: items and components containing thorium (December 2020)] (2020).

[37] Autodesk, Inc., "Autodesk Nastran User's Manual," 2018 (2018).

[38] The European Space Agency for the members of ECSS, [ECSS-E-ST-32-10C - Structural factors of safety for spaceflight hardware], The European Space Agency (ESA) (2009).

[39] ESA Education Office, "Fly Your Satellite': Design Specification Version 2.5, (ESA) Education," Rev. 2.5 (04 2018). Rev. 2.5, ESA-DG-SET-2018-1553.

[40] JAXA, "JEM Payload Accommodation Handbook - Vol. 8 - Small Satellite Deployment Interface Control Document," standard, Japan Aerospace Exploration Agency (JAXA) (Jan. 2015).

[41] NanoRacks, "NanoRacks CubeSat Deployer (NRCSD) Interface Control Document," standard, NanoRacks, 18100 Upper Bay Road, Suite 150, Houston, TX 77058, (815) 425-8553 (December 2013).

[42] The European Space Agency for the members of ECSS, [ECSS-Q-ST-70-02C - Thermal vacuum outgassing test for the screening of space materials], The European Space Agency (ESA) (2008).

[43] The European Space Agency for the members of ECSS, [ECSS-E-ST-10-03A - Space engineering: Thermal control general requirements], The European Space Agency (ESA) (2008).

[44] Zhan, S., Ahmad, I., Heuermann-Kuehn, L. E., and Baumann, J., "Dendritic Growth on the Die Under Hermetic High Temperature Operation," in [2010 Prognostics and System Health Management Conference], $1-6(2010)$.

[45] Otte, A. N., Garcia, D., Nguyen, T., and Purushotham, D., "Characterization of three high efficiency and blue sensitive silicon photomultipliers," Nuclear Instruments and Methods in Physics Research Section A: Accelerators, Spectrometers, Detectors and Associated Equipment 846, 106-125 (Feb 2017).

[46] Semiconductor Components Industries, LLC, ., "J-Series SiPM Sensors: Silicon Photomultipliers (SiPM), High PDE and Timing Resolution Sensors in a TSV Package, Rev6," (2018).

[47] Doyle, M., Dunwoody, R., Finneran, G., Murphy, D., Reilly, J., Thompson, J., Walsh, S., and et al., "Mission Testing for Improved Reliability of CubeSats," International Conference on Space Optics (2021). 